\title{
Applications of Abundance Data and Requirements for Cosmochemical Modeling
}

\author{
H. Busemann ${ }^{1}$, W. R. Binns ${ }^{2}$, C. Chiappini ${ }^{3}$, G. Gloeckler ${ }^{4}$, P. Hoppe ${ }^{5}$, D. \\ Kirilova $^{6}$, R. A. Leske ${ }^{7}$, O. K. Manuel ${ }^{8}$, R. A. Mewaldt ${ }^{7}$, E. Möbius ${ }^{9}$, R. Wieler ${ }^{10}$, \\ R. C. Wiens ${ }^{11}$, R. F. Wimmer-Schweingruber ${ }^{1}$ and N. E. Yanasak ${ }^{7}$ \\ ${ }^{1}$ Physikalisches Institut, University of Bern, Sidlerstr. 5, 3012 Bern, Switzerland \\ ${ }^{2}$ McDonnell Center for the Space Sciences, Washington University, St. Louis, MO, USA \\ ${ }^{3}$ Observatory of Trieste, Trieste, Italy \\ ${ }^{4}$ Department of Physics, University of Maryland, College Park, USA \\ ${ }^{5}$ Cosmochemistry Department, Max-Planck-Institute for Chemistry, Mainz, Germany \\ ${ }^{6}$ Institute of Astronomy, Bulgarian Academy of Sciences, Sofia, Bulgaria \\ ${ }^{7}$ Space Radiation Laboratory, California Institute of Technology, Pasadena, CA, USA \\ ${ }^{8}$ Department of Chemistry, University of Missouri, MO, USA \\ ${ }^{9}$ Department of Physics, University of New Hampshire, Durham, NH, USA \\ ${ }^{10}$ Institute for Isotope Geology and Mineral Resources, ETH Zürich, Zürich, Switzerland \\ ${ }^{11}$ Space and Atmospheric Sciences, MS D466, Los Alamos National Laboratory, Los Alamos, NM 87545, USA
}

\begin{abstract}
Understanding the evolution of the universe from Big Bang to its present state requires an understanding of the evolution of the abundances of the elements and isotopes in galaxies, stars, the interstellar medium, the Sun and the heliosphere, planets and meteorites. Processes that change the state of the universe include Big Bang nucleosynthesis, star formation and stellar nucleosynthesis, galactic chemical evolution, propagation of cosmic rays, spallation, ionization and particle transport of interstellar material, formation of the solar system, solar wind emission and its fractionation (FIP/FIT effect), mixing processes in stellar interiors, condensation of material and subsequent geochemical fractionation. Here, we attempt to compile some major issues in cosmochemistry that can be addressed with a better knowledge of the respective element or isotope abundances. Present and future missions such as Genesis, Stardust, Interstellar Pathfinder, and Interstellar Probe, improvements of remote sensing instrumentation and experiments on extraterrestrial material such as meteorites, presolar grains, and lunar or returned planetary or cometary samples will result in an improved database of elemental and isotopic abundances. This includes the primordial abundances of $\mathrm{D}$, ${ }^{3} \mathrm{He},{ }^{4} \mathrm{He}$, and ${ }^{7} \mathrm{Li}$, abundances of the heavier elements in stars and galaxies, the composition of the interstellar medium, solar wind and comets as well as the (highly) volatile elements in the solar system such as helium, nitrogen, oxygen or xenon.
\end{abstract}

\section{INTRODUCTION}

This manuscript is the résumé of a working group at the joint SOHO-ACE workshop held in Bern, Switzerland, in March 2001. The goal of this working group was "to determine the importance of various elements from the point of view of discerning different models that address questions such as solar-system formation, stellar and Big Bang nucleosynthesis, and chemical evolution of the Galaxy". We were asked to identify elements, which may serve as good references or indicators for the key physical processes involved. Certainly, we cannot provide a complete overview of all questions in the various disciplines relevant to this goal. Therefore, we will follow the expertise of the working group members and discuss some of the most crucial questions in cosmochemistry that can and should be answered in the near future through improvements in instrumentation, observational techniques, theory, and with new dedicated missions.

CP598, Solar and Galactic Composition, edited by R. F. Wimmer-Schweingruber (C) 2001 American Institute of Physics 0-7354-0042-3/01/\$18.00 
We will start with issues concerning the formation and composition of the solar system, and then address the composition of the (local) interstellar medium (L)ISM and of galactic cosmic rays (GCR). While the solar system represents a sample of galactic matter from 4.5 billion years ago, the LISM is a current and local sample of our Galaxy. Both samples provide benchmarks for models of galactic chemical evolution (GCE) from the Big Bang until today. GCRs represent another sample of the current Galaxy from a wide range of distances that shows some additional characteristics of high-energy interactions. With this paper, we aim to contribute to the interdisciplinary discussions between planetary scientists, solar physicists, cosmochemists, and astrophysicists.

\section{SOLAR SYSTEM ABUNDANCES}

\section{Highly Volatile Elements}

Absorption-line spectra of the solar photosphere and laboratory-based analyses of the most primitive meteorites, the CI (Ivuna-type) carbonaceous chondrites, yield solar-system abundances of the elements $(1,2)$. For most elements, the agreement of these data sets is $\sim 10 \%$ or better (Figure 1). However, meteorites do not represent solar abundances of the light or most volatile elements $\mathrm{H}, \mathrm{Li}, \mathrm{Be}, \mathrm{C}, \mathrm{N}, \mathrm{O}$, and the noble gases. The reasons are nucleosynthesis processes in the Sun's interior and the incomplete condensation during formation of the first solid matter in the solar system, respectively (2). Among the light elements, only the meteoritic abundance of boron agrees with the value recently determined in the photosphere (3). Therefore, even relatively imprecise measurements (compared to the precision usually obtained from meteorites) of all the mentioned elements in the Sun and the solar wind provide cosmochemically important information. The solar system isotopic composition of these elements is relatively poorly known. The light elements in meteorites are particularly subject to isotopic fractionation from originally solar system composition due to their volatility, the large relative mass differences of their isotopes and their chemical reactivity (4). It might well be that the isotopic composition of the noble gases in meteorites are not representative of the solar system at all (5), because the meteorite parent bodies or precursor planetesimals might never have incorporated these gases, in contrast to the much heavier planets which could have gravitationally captured gas from the nebula (6 and references therein, see also for alternative trapping mechanisms). The Sun and the gaseous giant planets Jupiter and Saturn, which formed only relatively small cores and hence remain largely undifferentiated, might represent isotopically undisturbed solar system composition or nearly so, although Jupiter appears to have a more evolved atmosphere, possibly of cometary origin (7).

The protosolar He abundance as well as its value in the present-day convective zone can be precisely determined by solar modeling and helioseismology (e.g., 8). However, for the solar $\mathrm{Ne}, \mathrm{Ar}, \mathrm{Kr}$, and $\mathrm{Xe}$ abundances, we must rely on extrasolar sources, analyses of implanted solar wind (SW) in lunar soils, and the systematics of s-process nucleosynthesis, which leads to rather large uncertainties of $15-25 \%$ ( 1 , 2 ). The abundance of these elements is important to assess the fractionation in the upper solar atmosphere according to first ionization potential or first ionization time (the so-called FIP/FIT effect, the relative enrichment of elements with FIP below $\sim 10 \mathrm{eV}$ in the low speed solar wind relative to photospheric abundances and high-FIP element abundances, e.g., 9 and references therein), compositional differences between the solar wind and solar energetic particles (SEP), temporal variability of the solar wind, or possible fractionation upon trapping in lunar soil (e. g., $10,11)$. Most solar wind noble gas isotopic ratios as derived from measurements of implanted solar wind in lunar material have stated precisions of $1 \%$ or better (12 and references therein) but better values are needed especially for the less abundant light $\mathrm{Kr}$ and $\mathrm{Xe}$ isotopes (see below). The Apollo Solar Wind Composition experiment (13) and space missions such as Ulysses (14) and SOHO $(15,16)$ also provided isotopic ratios for $\mathrm{He}-\mathrm{Ar}$ in the solar wind. Higher precision data from future missions, e.g., Genesis, are required, however, to test whether the values derived from lunar samples may be affected by isotopic fractionation upon or after trapping.

Solar elemental abundances of $\mathrm{C}, \mathrm{N}$, and $\mathrm{O}$ are believed to be known to within 8-12\%, comparable with the estimated accuracy for most elements in CI chondrites $(1,2)$. Their isotopic ratios, however, are not sufficiently well known. We will discuss below how a more precise solar oxygen isotopic composition is of high importance with respect to studies of the homogeneity of the solar nebula as well as for an improved understanding of the GCE. Even more controversial is the solar ${ }^{15} \mathrm{~N} /{ }^{14} \mathrm{~N}$ ratio, as we will discuss below. 


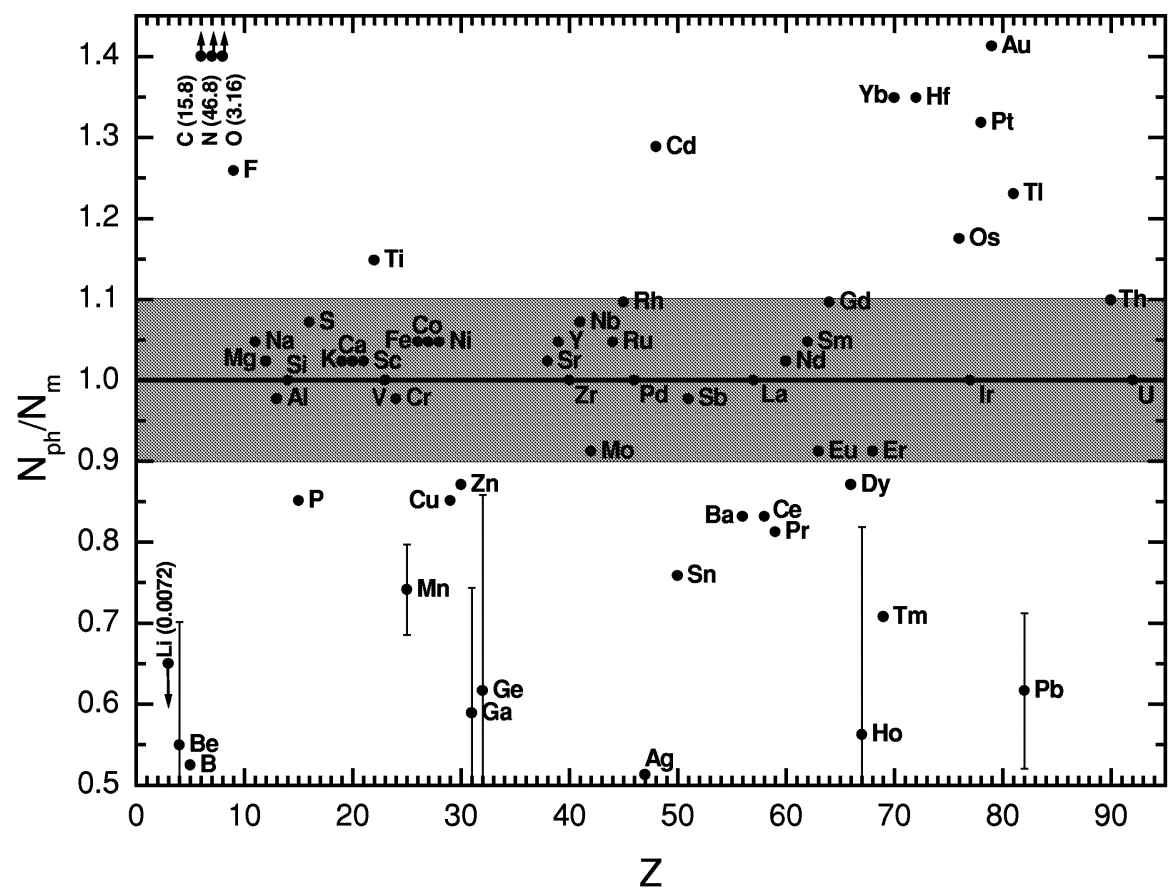

FIGURE 1. Comparison of solar photospheric $\left(\mathrm{N}_{\mathrm{ph}}\right)$ and meteoritic "CI" $\left(\mathrm{N}_{\mathrm{m}}\right)$ abundances (2). For most of the heavy elements, the abundances agree within $10 \%$. Error bars are shown if they do not overlap with the range $\mathrm{N}_{\mathrm{ph}} / \mathrm{N}_{\mathrm{m}}=0.9-1.1$. Only $\mathrm{Mn}$ and $\mathrm{Pb}$ show significant deviations not covered by the uncertainties. Figure after (2) with kind permission from Springer-Verlag, Heidelberg.

The isotopic signature of $\mathrm{Li}$ and $\mathrm{B}$ in the solar system, which is influenced by nuclear reactions, both in the upper solar atmosphere and in early condensed material, is discussed in detail elsewhere (e.g., 17, 18 and references therein).

Precise data on the abundances of the volatile elements in the solar wind will be essential to improve the knowledge of fractionation mechanisms that mask the solar source composition. Measurements of isotopic abundances of other, more refractory, elements in the solar wind such as $\mathrm{Mg}$ (15) will help to address this issue. Many of the future efforts, however, focus on the determination of the elemental and isotopic abundances of $\mathrm{N}, \mathrm{O}$, and the noble gases. Future missions to comets (19) will obtain precise abundances of the volatile elements that should fill some of the gaps left by meteorites, because comets condensed at large distances to the Sun at low temperatures and thus contain more pristine material than that found in meteorites (20). In the following, we will discuss some of the volatile elements and the issues they address in more detail.

\section{The Genesis Mission and Solar Oxygen}

The Genesis mission, launched on August 8, 2001, will collect solar wind particles for about two years in a variety of targets that will be analyzed on Earth. This will significantly improve the precision of solar wind isotope measurements for many elements. The highest priority objective is a precise determination of the oxygen isotopic composition in the solar wind as discussed below. This is a good starting point to discuss the importance of accurate solar abundance values for an improved understanding of solar system formation and current solar processes. These include nebular mixing issues needed to understand the differences among planetary compositions and planetary atmospheres. A further high priority objective is the measurement of the isotopic composition of nitrogen and the noble gases in the solar wind. These issues will be discussed in detail in the following paragraphs. 




FIGURE 2. Solar oxygen isotopic compositions predicted by the nebular mixing model (24) and by the non-mass-dependent fractionation model (29). $\mathrm{X}$ and $\mathrm{Y}$-axes give ${ }^{18} \mathrm{O}$ and ${ }^{17} \mathrm{O}$ enrichments relative to standard mean ocean water in \%o. Present solar composition uncertainties are on the order of $\pm 200 \%$ or or ${ }^{18} \mathrm{O} /{ }^{16} \mathrm{O}$, while the solar ${ }^{17} \mathrm{O} /{ }^{16} \mathrm{O}$ ratio is completely unconstrained $(25$ 27). With the Genesis mission, it is hoped to measure solar wind oxygen isotopes to $\pm 1 \%$, which is sufficient to distinguish between the models. $\mathrm{OC}=$ ordinary chondrites; $\mathrm{R}=\mathrm{R}$ (Renazzo-type) chondrites; $\mathrm{CAI}=$ calcium-aluminum-rich inclusions. "Magnetites" refers to individual magnetite grains in unequilibrated ordinary chondrites.

The Genesis mission results will also help to determine nuclear processes operating on the surface of the Sun and in solar system precursor materials, and solar-specific processes operating either early or later in the solar system history. An example of the latter is a comparison of heavy and light elements relative to CI chondrites indicative of solar gravitational settling (21). Examples of the former are $\mathrm{Li}, \mathrm{Be}, \mathrm{B}$ isotopic and elemental abundances, which will help to understand the history of the solar convection zone (e.g., 22). Examples of objectives addressing nuclear processes are, e.g., the solar $\mathrm{F}$ abundance as a measure of integrated spallation production through time and comparisons of $\mathrm{Kr}$ and $\mathrm{Xe}$ abundances to meteoritic abundances of neighboring elements in the periodic table as a measure of solid-gas fractionation during formation of the Sun (23).

The three oxygen isotopes show clear differences between various solar system bodies of a few permil up to several percent (Figure 2). So far, we have measurements for Earth, Moon, Mars, Vesta, (via meteorites), ordinary and carbonaceous chondrites, and miscellaneous differentiated meteorites (24). Measurements of the outer planet or Hermean oxygen compositions, however, are missing. Oxygen compositions of the Sun (e.g., 25, 26, and 27, this volume) and comets (28) are known only at the $~ 10$ $20 \%$ level, and do not include ${ }^{17} \mathrm{O}$.

At present, we do not understand the oxygen isotopic variations. One theory (e. g., 29) implies that non-mass-dependent fractionation acted on an initially homogeneous hot solar nebula reservoir to produce ${ }^{16} \mathrm{O}$ enrichments in the initially crystallizing refractory materials. The other theory (e.g., 24 and references therein), suggests that nebular mixing between an ${ }^{16} \mathrm{O}-$ enriched solid composition and a ${ }^{16} \mathrm{O}$-poor nebular gas produced the variety of compositions seen now. In its simple form, the nebular mixing model predicts for the Sun a significantly more ${ }^{16} \mathrm{O}$-depleted composition than the non-mass-dependent model, for which a composition essentially identical to the Earth, Moon, and Mars is inferred. A measurement of $\pm 0.1 \%$ in ${ }^{17} \mathrm{O} /{ }^{16} \mathrm{O}$ and ${ }^{18} \mathrm{O} /{ }^{16} \mathrm{O}$ can distinguish between these (Figure 2,30). This is a reduction by a factor of more than 100 from the current measurement uncertainties for solar oxygen (25-27). Within the confines of the nebular mixing model, there are also ways in which ${ }^{18} \mathrm{O} /{ }^{16} \mathrm{O}$ in the Sun could be significantly larger than the simplistic prediction. If so, a measurement at the $\sim 1 \%$ level will suffice to distinguish between these models, but further precision will give insight into nebular processes such as possible fractionation 
between nebular $\mathrm{CO}$ and $\mathrm{H}_{2} \mathrm{O}$ gas during meteorite formation or solid/gas enrichment in the meteoriteforming region (30).

\section{Nitrogen in the Sun and the Solar System}

Along with $\mathrm{H}, \mathrm{C}, \mathrm{O}$, and the noble gases, nitrogen belongs to the incompletely condensed elements in meteorites whose elemental abundance and isotopic composition cannot therefore be deduced from CI chondrite data. Unlike for the noble gases, it has also proven very difficult to deduce the isotopic composition of solar nitrogen from analyses of samples that were exposed to the solar wind. The currently used solar system abundance compilations (e. g., 1, 2) therefore adopt the ${ }^{15} \mathrm{~N} /{ }^{14} \mathrm{~N}$ ratio in the terrestrial atmosphere $\left(3.68 \times 10^{-3}\right)$ as a solar system standard, although differences between solar and terrestrial noble gases illustrate that this is not necessarily correct.

A crucial but ill-understood observation is that the nitrogen isotopic composition trapped in lunar soils varies by up to $35 \%$ in different bulk samples and in different extraction steps of an individual soil $(31,32)$. Classically, these observations have been interpreted to indicate a secular increase of the ${ }^{15} \mathrm{~N} /{ }^{14} \mathrm{~N}$ ratio in the solar wind from about $2.9 \times 10^{-3}$ several Gyrs ago to perhaps up to $4.1 \times 10^{-3}$ for the recent solar wind (33). This interpretation implies that essentially all trapped nitrogen in lunar soil samples is from the solar wind. There are two major problems with the hypothesis of a secular change of the solar wind nitrogen composition. First, there is no generally accepted process for providing such a fractionation (34). For instance, isotopic fractionation in the solar wind has been shown to be small (at most a few \%/amu) based on measurements of the isotopes of the refractory elements $\mathrm{Mg}$ and $\mathrm{Si}(15,35$, and 36, this volume, and references therein). Postulating an increased solar activity in the past can probably not explain the isotopic behavior either. Measurements of the $\mathrm{Mg}$ and $\mathrm{Si}$ isotopic compositions in coronal mass ejections (CME), the most dramatic manifestation of increased solar activity, show no fractionation (35). Second, the abundance ratio $\mathrm{N} /{ }^{36} \mathrm{Ar}$ in lunar samples is about an order of magnitude higher than the respective solar wind ratio. The latter observation may indicate preferential loss of solar wind-implanted ${ }^{36} \mathrm{Ar}$ relative to $\mathrm{N}$, e.g., by diffusion, but analyses of single lunar dust grains indicate that this is probably not the case, implying that nitrogen in the lunar regolith has a predominantly non-solar source (37). In summary, the nitrogen isotopic composition as deduced from lunar regolith samples is highly controversial. A value of $(3.82 \pm 0.02) \times 10^{-3}$ has been proposed from analyses of two relatively recently irradiated samples (38), whereas ion-probe measurements on single grains thought to contain relatively little non-solar nitrogen yielded a value of $<2.79 \times 10^{-3}$ (39), and multi-step analyses of single grains by incremental heating support such an isotopically light composition (40). These workers attribute the variable ${ }^{15} \mathrm{~N} /{ }^{14} \mathrm{~N}$ ratios in lunar samples to a variable admixture of a non-solar component possibly of meteoritic origin (41, this volume).

Comparing the various lunar estimates with the direct isotopic analysis of solar wind nitrogen by the CELIAS/MTOF instrument on SOHO (42) and a value for the Jovian atmosphere obtained by the Galileo probe (43) does not help to resolve the conflict. The SOHO value of $5.0_{-1.1}^{+1.9} \times 10^{-4}$ is compatible within its large uncertainty with the value by $\mathrm{Kim}$ et al. (38), whereas the Galileo probe value of $(2.3 \pm 0.3) \times 10^{-3}$ (43) and the value obtained with ISO-SWS $\left(1.9_{-1.0}^{+0.9}\right) \times$ $\left.10^{-3}, 44\right)$ are consistent with the isotopically light value advocated by Hashizume and coworkers (39, Figure $3)$. The latter comparison assumes that the Jupiter value is representative for the (proto) solar composition. Owen et al. (43) argue that this is the case. In HCN in interstellar clouds, ${ }^{15} \mathrm{~N}$ is enriched by up to $30 \%(45)$, but $\mathrm{N}$ in Jupiter is derived from protosolar $\mathrm{N}_{2}$. The ${ }^{15} \mathrm{~N} /{ }^{14} \mathrm{~N}$ ratio in $\mathrm{HCN}$ in comet Hale-Bopp's coma is indeed about $30 \%$ higher than the Galileo value (Figure 3, see discussion in 43).

Given this situation, it becomes obvious why a precise determination of the nitrogen isotopic composition in the solar wind is one of the two highest-priority goals of the upcoming Genesis mission. Hopefully, this will not only allow us to come closer to a solution of the conundrum of the origin of nitrogen in the lunar regolith, but also yield important clues as to the relation between giant planets and the Sun. A well defined nitrogen isotopic composition in the Sun is also needed for an improved understanding of the highly variable composition of this element in the various meteorite classes, which show ${ }^{15} \mathrm{~N} /{ }^{14} \mathrm{~N}$ ratios in the range $(3.2-9.5) \times 10^{-3}$ (46, Figure 3$)$. It remains unclear to what extent this variability in meteorites reflects a large isotopic heterogeneity in the early solar system or fractionation processes that might have occurred during condensation or later in the meteorite parent bodies. 


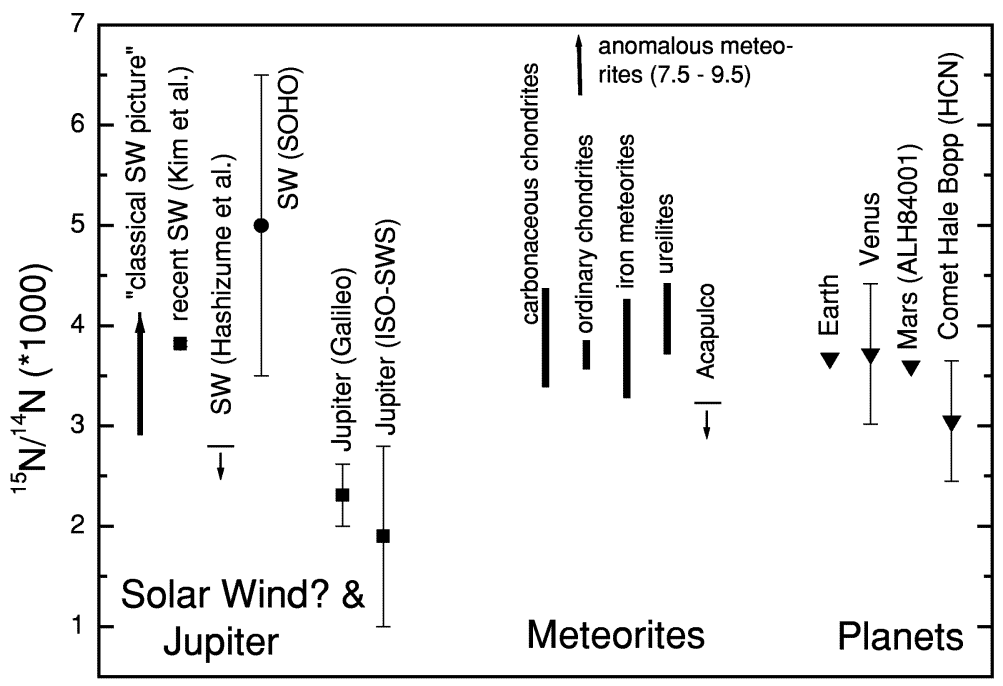

FIGURE 3. Various published ${ }^{15} \mathrm{~N} /{ }^{14} \mathrm{~N}$ ratios for the solar wind and Jupiter's atmosphere compared with the nitrogen composition of meteorites, planets, and comet Hale-Bopp. The "classical SW picture" postulates a secular increase of ${ }^{15} \mathrm{~N} /{ }^{14} \mathrm{~N}$ in the solar wind over the past several Gyrs (33). Data sources for SOHO, Galileo probe, and ISO-SWS values see text. Venus, Mars, Hale-Bopp, and meteorite values are taken from references $(43,46)$.

\section{(Proto) Solar and Cometary Xenon Isotopic Composition}

The chemically inert noble gases are important tracers to understand physical processes that formed the planets and their atmospheres. Distinct models discuss the noble gases in the terrestrial planets (e. g., $47,48,49,50)$. Their atmospheres are strongly depleted in the light elements relative to the heavier elements and solar composition. The surprisingly uniform trapped primordial noble gas component in meteorites yields a similar element composition (51). However, distinct isotopic signatures in the atmospheres of Earth and Mars (they are similar to each other and "isotopically heavier" than meteoritic noble gases) and "missing $\mathrm{Xe}$ " in the atmospheres exclude a common precursor of meteoritic and atmospheric noble gases (49). As we will see below, the $\mathrm{Xe}$ isotopic composition of these atmospheres cannot be explained by simple fractionation of solar noble gases, although the interior of the Earth $(6,52$, $53)$ and possibly Mars $(54,55)$ contain solar-like noble gases. Xenon, with nine stable isotopes, is very suitable to discuss many of the processes that led to the present-day compositions of the atmospheres such as fission and decay, impact-induced and hydrodynamic loss, as well as additions of cometary and meteoritic gas. We will discuss two models for the origin of terrestrial and Martian atmospheric Xe in the following: (i) Pepin's model suggests fractionation of $a$ hypothetical primordial component dubbed U-Xe (56). This fractionation is the result of the preferential loss of the light species relative to a heavier one upon an early hydrodynamic escape of a dense primordial atmosphere and can explain the "planetary" element pattern. If one fractionates solar Xe in a way that the isotopic ratios ${ }^{124 / 126 / 128} \mathrm{Xe} /{ }^{130} \mathrm{Xe}$ are in agreement with the terrestrial atmosphere, the heavier isotopes ${ }^{134 / 136} \mathrm{Xe}$ are already overabundant relative to the observed patterns (Figure 4a). This would thus not allow for contributions of ${ }^{134 / 136} \mathrm{Xe}$ from fission of ${ }^{238} \mathrm{U}$ and ${ }^{244} \mathrm{Pu}$ in the Earth's interior. To account for this fission $\mathrm{Xe}$, the primordial U-Xe needs to have lower abundances of ${ }^{134 / 136} \mathrm{Xe}$ than the Sun (56).

Interestingly, fits on achondrite and chondrite Xe isotopic data seem to point to a common endmember component very similar to this independently deduced component. The ubiquitous and relatively uniform meteoritic Xe ("Xe-Q", 51) can be obtained by fractionation of $\mathrm{U}-\mathrm{Xe}$ and addition of ${ }^{134} \mathrm{Xe}$ and ${ }^{136} \mathrm{Xe}$ ("Xe-H"), whereas solar Xe is unfractionated U-Xe plus significant amounts of Xe-H (Figure 4b). U-Xe, although a potential precursor of $\mathrm{Xe}$ in the terrestrial atmosphere, has not unambiguously been observed (57). It is unclear where and how the fractionation of $\mathrm{U}-\mathrm{Xe}$ into $\mathrm{Xe}-\mathrm{Q}$ in the different meteorite classes occurred and why the Sun does not contain the putative primitive U-Xe. 

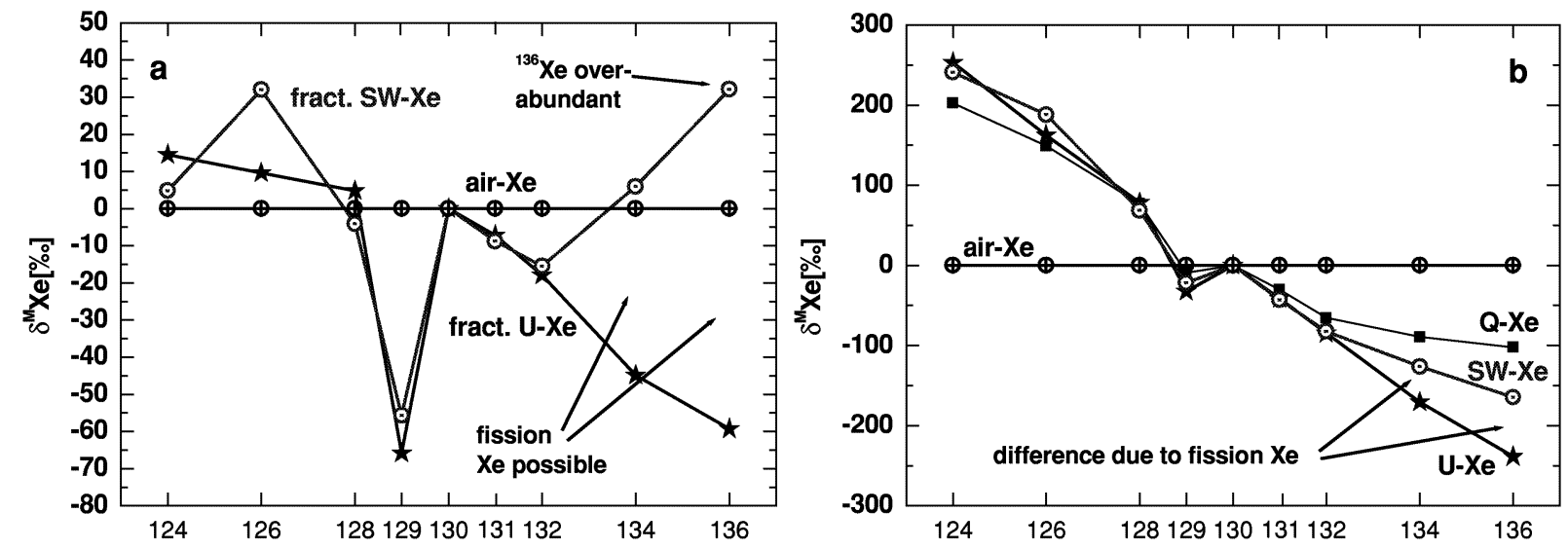

FIGURE 4. Isotopic composition of solar wind and U-Xe, plotted as deviation from terrestrial atmospheric Xe in \%o. Pepin suggested that the terrestrial atmosphere Xe could be deduced by fractionation of a solar-type Xe component (56). a. Fractionated solar wind already yields an excess of ${ }^{134} \mathrm{Xe}$ and ${ }^{136} \mathrm{Xe}$, if the light isotopes of both components roughly correspond. Addition of fission Xe from the interior would increase the discrepancy. b. U-Xe, which is solar wind Xe depleted in the heavy isotopes, accounts for contributions of fission Xe from the interior. Fractionated and supplemented by heavy Xe, it also reproduces the common trapped Xe-Q component in meteorites. Data given in $(6,51$, and 56$)$.

(ii) If hydrodynamic escape is the reason for the similarly fractionated composition of both the Martian and terrestrial atmosphere, then this similarity must be coincidental, because of the different masses of these planets. Otherwise, atmospheric Xe on Mars and Earth may have their root in a common fractionated source (58). A cometary origin has been proposed by Owen and coworkers (48). They suggested that $\mathrm{Ar}, \mathrm{Kr}$, and $\mathrm{Xe}$ in the atmospheres of the terrestrial planets are a mixture of an internal component and a contribution from impacting comets that must have formed at a temperature of about $50 \mathrm{~K}$.

One important step to clarify this issue is to measure the $\mathrm{Xe}$ isotopes in comets and the outer planets. Most indicative are the ratios ${ }^{134} \mathrm{Xe} /{ }^{132} \mathrm{Xe}$ and ${ }^{136} \mathrm{Xe} /{ }^{132} \mathrm{Xe}$. Uncertainties of $\sim 1 \%$ are needed, however, to decide whether comets and the gaseous planets contain meteoritic or solar wind Xe. Ratios within $2 \%$ would resolve U-Xe and SW-Xe. For comparison, the Galileo probe measured the respective $\mathrm{Xe}$ ratios in Jupiter's atmosphere with uncertainties of $\sim 10 \%(59,60)$. Measurements of the solar wind $\mathrm{Xe}$ as trapped by the Genesis mission (see above) with expected uncertainties $<1 \%$ will allow refining the models. At present the ratios ${ }^{124} \mathrm{Xe} /{ }^{132} \mathrm{Xe}$ and ${ }^{126} \mathrm{Xe} /{ }^{132} \mathrm{Xe}$ in the solar wind are known to within 2$4 \%$. So far, only lunar soil has yielded solar wind Xe isotopic ratios $(61,62)$. Xe data from Genesis will facilitate a comparison of ancient with present solar wind, and check for possible fractionation processes during trapping on the moon.

\section{Presolar Grains and Solar System Si- and O-Isotopic Ratios}

Meteorites contain refractory grains of stardust believed to originate from stellar outflows and supernova ejecta. Large isotopic anomalies, e.g., of $\mathrm{Ne}$ and $\mathrm{Xe}$, led to the identification of several types of presolar grains and provide important clues on stellar nucleosynthesis processes and galactic evolution (63, 64 and references therein, see also 65 , this volume). Presolar $\mathrm{SiC}$ is the major carrier of $\mathrm{Si}$ among meteoritic stardust. Because its average ${ }^{29} \mathrm{Si} /{ }^{28} \mathrm{Si}$ and ${ }^{30} \mathrm{Si} /{ }^{28} \mathrm{Si}$ ratios are a few percent higher than the solar system ratios (66), other yet unidentified presolar Sibearing mineral types with isotopically light $\mathrm{Si}$ may have contributed to the Si budget of the solar system. Potential candidates are silicates that are believed to be a major circumstellar condensate (67). The search for presolar silicates is complicated by the fact that the separation of presolar minerals from meteorites relies on the use of chemicals that destroy silicates. The successful identification of presolar silicates requires the use of non-destructive separation techniques, the in situ search in meteoritic thin sections, or the analysis of cometary samples which will become available from the STARDUST mission (19). The latter samples are of particular interest as comets represent the most 
primitive solar system matter, thus being a potentially rich source of yet unidentified presolar mineral types.

Similarly, the average ${ }^{17} \mathrm{O} /{ }^{16} \mathrm{O}$ ratio of presolar $\mathrm{O}$ bearing minerals, mainly corundum and to some extent graphite, is higher than solar (see 63, 64), emphasizing the need for a search for ${ }^{16} \mathrm{O}$-rich presolar grains. This aspect is also closely related to the puzzle of the low abundance of oxide grains from supernovae ( $\mathrm{SN}$ ) among meteoritic stardust because such grains are expected to be predominantly ${ }^{16} \mathrm{O}$-rich. It has been argued that SN oxide grains have remained largely undetected among meteoritic stardust because they are smaller than those from red giant stars, which are rich in ${ }^{17} \mathrm{O}$ and which make up the major fraction of presolar oxide grains (68). A systematic search for smaller oxide grains as well as a search for yet unidentified mineral types may allow finding an answer on this question.

\section{The ${ }^{53} \mathrm{Cr} /{ }^{52} \mathrm{Cr}$ Ratio as Indicator for the Formation Region of Planetary Bodies?}

Several short-lived (half-lives of $\sim 10^{6}$ yrs) now extinct radionuclides have been incorporated into or produced in situ in early solar system material. The decay leads to enrichments of daughter nuclides and thus allows the relative chronology of early solar system processes, if the initial daughter/reference nuclide ratio is known. The short-lived ${ }^{53} \mathrm{Mn}$ decays to ${ }^{53} \mathrm{Cr}$. The ${ }^{53} \mathrm{Cr} /{ }^{52} \mathrm{Cr}$ ratio of planetary samples (Earth \& Moon, meteorites from Mars ("SNCs" - shergottites, nakhlites, chassignites) and Vesta ("HEDs" howardites, eucrites, diogenites) apparently correlates with heliocentric distance of the place of formation (69, see also 65 , this volume). If this is generally true, then the initial ${ }^{53} \mathrm{Mn}$ abundance could be used to constrain the place of formation of solar system samples of unknown origin. However, the radial heterogeneity of the initial ${ }^{53} \mathrm{Mn}$ in the solar nebula has been called into question (70). New data on chondrules in unequilibrated meteorites suggest volatilitycontrolled variation of $\mathrm{Mn}$ and $\mathrm{Cr}$ in the nebula instead. In this respect, it would be of particular interest to measure ${ }^{53} \mathrm{Cr} /{ }^{52} \mathrm{Cr}$ in other inner solar system objects, e.g., the Sun (or solar wind) or Mercury. The required accuracy is about $10 \mathrm{ppm}$, which cannot be achieved by space missions planned (e.g., to Mercury) in the foreseeable future, but would require sample return missions.

\section{A Sun Consisting Mainly of Fe and Ni?}

One co-author (O. M.) favors a non-standard model of solar system formation and composition in which the Sun formed on a supernova core. We discuss this model here although it has not gained acceptance in the community. The empirical comparison of noble gas isotope abundances in solar-wind-rich lunar soil and the meteorite Allende (see Figure $4 \mathrm{~b}$ for the Xe isotopic composition in solar wind and meteorites) led Manuel and Hwaung (71 and references therein) to suggest intra-solar diffusion that follows a massdependent power law to explain differences between meteorites and the solar wind. This diffusion should result in a solar surface enriched in the light elements $\mathrm{H}$ and $\mathrm{He}$, as observed, whereas the most abundant elements in the solar interior are $\mathrm{Fe}, \mathrm{Ni}, \mathrm{O}, \mathrm{Si}, \mathrm{S}, \mathrm{Mg}$, and $\mathrm{Ca}$. This solar composition then resembles that found in bulk meteorites.

The enrichment of the lighter (mass $\mathrm{m}_{\mathrm{L}}$ ) relative to the heavier $\left(\mathrm{m}_{\mathrm{H}}\right)$ isotope must also be discernible in the solar wind isotope composition of other elements. The power law describes the fractionation (f) with $\mathrm{f}=\left(\mathrm{m}_{\mathrm{H}} / \mathrm{m}_{\mathrm{L}}\right)^{4.5}$ and thus predicts, e.g., a $\mathrm{Mg}$ isotope fractionation of $\sim 20 \% / \mathrm{amu}$. The $\mathrm{Mg}$ concentration in lunar soils is much too high to detect solar wind $\mathrm{Mg}$, but in situ measurements of the isotopic composition of the refractory elements $\mathrm{Mg}$ and $\mathrm{Si}$ in the solar wind show that they are fractionated by at most $2 \% / \mathrm{amu}$ in the solar wind relative to terrestrial or meteoritic values $(15,35,36)$. In addition, the $\mathrm{Al} / \mathrm{Mg}$ ratio in the solar wind is indistinguishable from the solar system value obtained from meteorites (72), in contrast to the model by (71) that predicts a $\mathrm{Mg}$ enhancement of $70 \%$. Furthermore, this model will have to prove its capability to match all observational constraints from helioseismology, solar neutrino flux observations, and the average solar density of $\sim 1.41 \mathrm{~g} / \mathrm{cm}^{3}$.

\section{THE INTERSTELLAR MEDIUM}

The composition of the interstellar medium provides an important benchmark in the discussion of several cosmological and cosmochemical issues. It helps to decide whether the Sun's composition is unusual for this part of the Galaxy (73, this volume), and serves as reference composition for current galactic material and thus is essential for GCE models (see below). The composition of the dust and the gas in the ISM also serves as a baseline for the source of GCRs, thought to be mainly accelerated interstellar material (see below), and finally provides useful 
information for understanding Big Bang nucleosynthesis.

Optical and radio spectroscopy as well as direct measurements of infalling particles in the heliosphere provide elemental and isotopic abundances in the local interstellar medium (LISM). In the following, we will briefly review the state of satellite-based particle measurements, such as the detection of pickup ions and anomalous cosmic rays within the heliosphere. We will then discuss the prospects of future heliospheric missions to address these goals, culminating in an interstellar medium probe to beyond the heliosphere.

\section{Measurements of the Interstellar Medium by Pickup Ions}

Most pickup ions in the solar wind are particles originating from the interstellar medium that enter the heliosphere as neutral atoms. Subsequently, they become singly ionized and acquire energies in the range 0 to $4 \mathrm{E}_{\mathrm{Sw}}$, i.e. roughly $0-10 \mathrm{keV} / \mathrm{nuc}$ (The only exception is ${ }^{3} \mathrm{He}$ which is doubly ionized). Therefore, pickup ions provide an important source to our knowledge of both the composition of the Local Interstellar Cloud (LIC) as well as of the filtration processes occurring in the heliospheric interface region.

Element abundances of $\mathrm{H}, \mathrm{He}, \mathrm{N}, \mathrm{O}$, and $\mathrm{Ne}$ and the ${ }^{3} \mathrm{He} /{ }^{4} \mathrm{He}$ isotopic ratio have been determined (74, 75). Other heavy elements with low first ionization potential (FIP) are already mostly ionized in the interstellar medium and therefore do not enter the heliosphere. Pickup ions of low FIP elements, such as $\mathrm{C}$, have been found, but they apparently mainly originate from the "inner source" (most likely solarwind-loaded interplanetary dust in the solar system) and can be easily distinguished by their velocity distribution $(76,77)$.

Current knowledge of the elemental composition in the solar system and the LISM suggests only a slight, if any, overabundance of $\mathrm{N}$ and about an equal abundance of $\mathrm{O}$ and $\mathrm{Ne}$ in the LISM compared to the solar system (78, this volume). The uncertainties for these elements in both reservoirs are still very large. The uncertainty of elemental abundances in the LISM using pickup ions is at present $\sim 25 \%$ or more. An accuracy of a few percent is necessary to better constrain models of GCE (see below). Therefore, significant improvements in the accuracy of abundances are urgently needed. Improvements need to be made in two ways. First, the counting statistics have to be improved. Because the density of all interstellar neutrals, except $\mathrm{He}$, increases significantly between 1 and 3 to $10 \mathrm{AU}$, dedicated instruments with high resolution and large collection power on a spacecraft in a 1 by $3 \mathrm{AU}$ orbit are required to increase the accuracy of measurements to less than one percent. An "Interstellar Pathfinder" mission with such performance characteristics has recently been proposed (79). Second, measurements of, and models describing the physical state of the ISM and the characteristics of the interface region between the heliosphere and the LIC should be improved. This is necessary in order to increase the accuracy of estimates of the degree of ionization of the LIC gas and the fraction of elements bound in interstellar dust, both of which are required to obtain the elemental composition of the LIC from pickup ions. The density of interstellar neutral $\mathrm{H}, \mathrm{N}$, and $\mathrm{O}$ in the heliosphere is systematically reduced from the corresponding densities in the LIC by filtration in the heliospheric interface region (e.g., 80, 81). Using pickup ion observations, absorption measurements from nearby stars and a simple model of the interface region, constraints on the amount of filtration of $\mathrm{H}, \mathrm{N}$, and $\mathrm{O}$ and on the ionization fractions of $\mathrm{H}$ and $\mathrm{He}$ were obtained (78, this volume, 82$)$. More detailed probing of the filtration can be expected from direct observation of the interstellar neutral gas flow pattern in the inner heliosphere (e.g., 83). Nevertheless, the composition of low-FIP elements will remain inaccessible to in situ observations inside the heliosphere. To remove this ultimate restriction will require an "Interstellar Probe" mission into the neighboring interstellar medium (84).

Largely unaffected by filtration at the heliospheric boundary is the determination of isotopic composition of the LISM through in situ observations in the heliosphere. Especially important is the He isotopic composition, because it represents the present-day ${ }^{3} \mathrm{He}$ abundance in the LISM. In combination with the protosolar ${ }^{3} \mathrm{He}$ abundance, deduced from meteorites (85) and/or the Jovian atmosphere (59), it allows one to trace the evolution of the Galaxy over the past 4.56 Gyrs (see below). $\mathrm{A}{ }^{3} \mathrm{He} /{ }^{4} \mathrm{He}$ ratio of $2.48_{-0.62}^{+0.68} \times 10^{-4}$ has been determined from pickup ions observed with SWICS on Ulysses (86). This ratio is, within the large uncertainties, comparable with $1.7_{-0.42}^{+0.50} \times 10^{-4}$ measured in foils directly exposed to the inflowing neutral interstellar gas onboard the space station MIR (87, this volume). Both ${ }^{3} \mathrm{He} /{ }^{4} \mathrm{He}$ ratios are known to within $\sim 25 \%$. Desirable is an accuracy of $<5 \%$, because this ratio yields, in combination with the present-day solar wind value, measured during several missions, the very high priority protosolar $\mathrm{D} / \mathrm{H}$ ratio, which sets tight constraints on the baryon density of 
the universe. This example illustrates that more, and more precise, measurements of the LISM are extremely important. The recent re-determination of the ${ }^{3} \mathrm{He} /{ }^{4} \mathrm{He}$ ratio in the solar wind with SWICS on Ulysses, e.g., has already provided an averaged ${ }^{3} \mathrm{He} /{ }^{4} \mathrm{He}=(3.75 \pm 0.27) \times 10^{-4}$ in the outer convection zone (88).

Except for the ${ }^{3} \mathrm{He} /{ }^{4} \mathrm{He}$ ratio, all other isotopic ratios in the LISM measured with pickup ions are essentially unconstrained. The ${ }^{20} \mathrm{Ne} /{ }^{22} \mathrm{Ne}$ ratio is especially important, as it allows a comparison with the ratio obtained from anomalous cosmic rays (see below) and may help to understand the discrepancy between values obtained in meteorites, solar wind, and energetic particles. An "Interstellar Pathfinder"-like mission could provide the mass resolution and collecting power to achieve these goals (79).

\section{Anomalous Cosmic Rays as Probe of the Interstellar Medium}

The elements $\mathrm{H}, \mathrm{He}, \mathrm{C}, \mathrm{N}, \mathrm{O}, \mathrm{Ne}$, and $\mathrm{Ar}$ have been clearly detected in "anomalous cosmic rays" (ACR), which are called so, because their composition is neither solar nor similar to that in galactic cosmic rays (89). After ionization and pick up by the solar wind in the inner heliosphere, the formerly interstellar atoms are convected to the outer heliosphere, where they are accelerated at the termination shock to energies 1-100 MeV/nuc (89). Possibly, $\mathrm{Na}, \mathrm{Mg}, \mathrm{S}$, and Si have also been detected in ACRs $(90,91)$. However, their origin is not clear. All four elements have a low FIP $<10.4 \mathrm{eV}$ and should have a low neutral abundance in the interstellar medium (92).

Isotopically resolved measurements (especially at energies below $10 \mathrm{MeV} / \mathrm{nuc}$ ) are most important. For example, the upper limit on the ACR ${ }^{15} \mathrm{~N} /{ }^{14} \mathrm{~N}$ ratio is about 10 times larger than that adopted for the Sun (93, Figure 5a), since it is limited by GCR background. Thus, a future measurement of $\mathrm{N}$ isotopes with $50 \%$ uncertainty at energies $<10 \mathrm{MeV} /$ nuc, where the GCR background is less, would be desirable. The ${ }^{18} \mathrm{O} /{ }^{16} \mathrm{O}$ ratio however needs to be measured to within some $10 \%$, as the ratios deduced for the Sun and the ACR agree within a factor of two (93, Figure 5b). The ${ }^{20} \mathrm{Ne} /{ }^{22} \mathrm{Ne}$ isotopic ratio in ACRs has been found to match solar wind or meteoritic composition, with the former being more likely (Figure 5c, options "c" and "b", respectively).

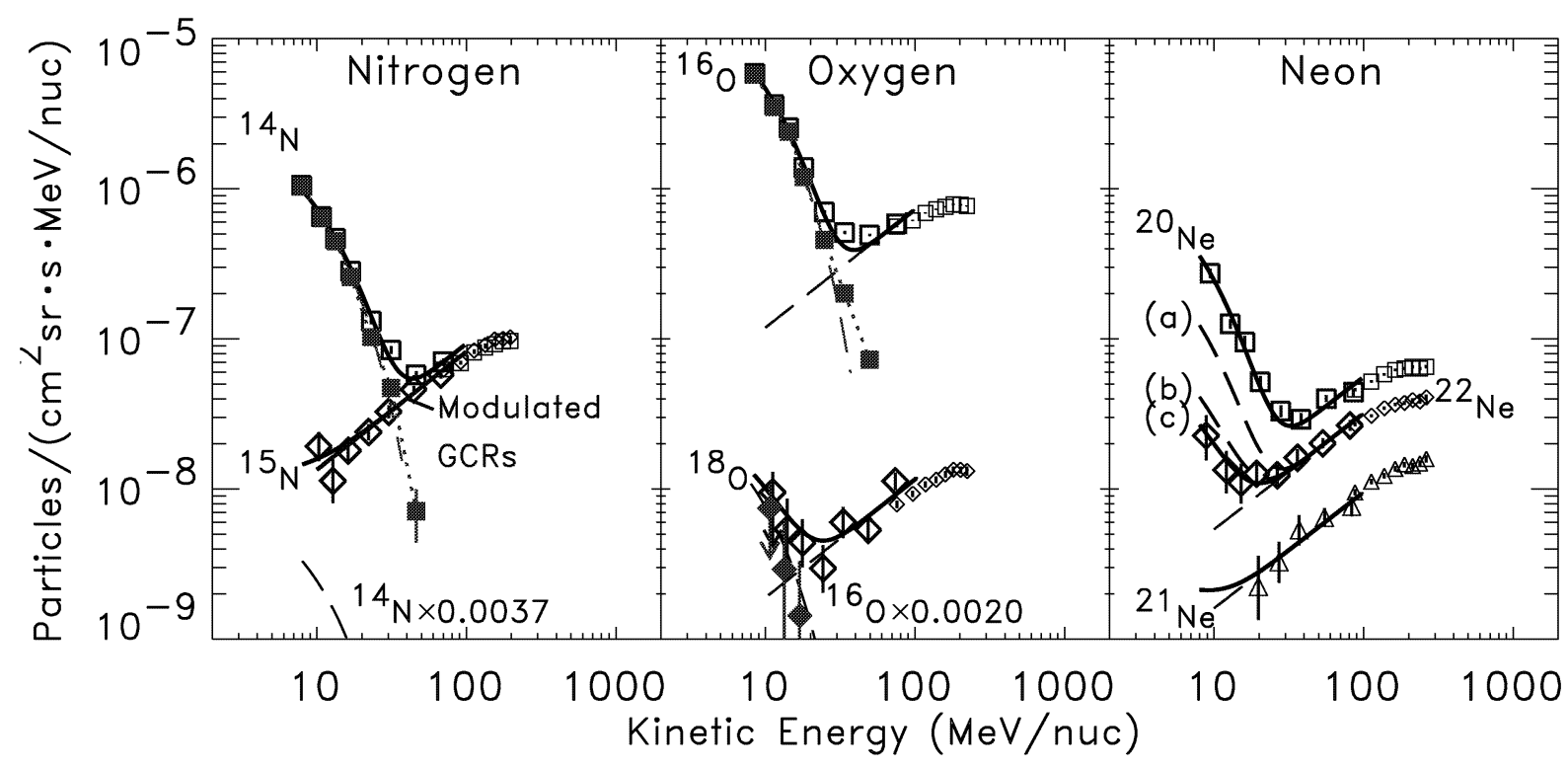

FIGURE 5. Isotopic composition of $\mathrm{N}, \mathrm{O}$, and $\mathrm{Ne}$ in the anomalous component of cosmic rays as measured with the Solar Isotope Spectrometer (SIS) on the Advanced Composition Explorer (ACE) (93). Open symbols are measured data points. The low energy excesses are due to anomalous cosmic rays. a) and b) Dashed lines are expected ${ }^{15} \mathrm{~N}$ and ${ }^{18} \mathrm{O}$ abundances, respectively, using ${ }^{14} \mathrm{~N}$ and ${ }^{16} \mathrm{O}$ abundances and assuming solar system composition. The figure indicates that any ${ }^{15} \mathrm{~N}$ in the ACRs is not detectable at these energies above the galactic cosmic ray (GCR) background. After subtracting this background, the remaining ACR ${ }^{18} \mathrm{O}$ (filled symbols) is consistent (within a factor of $\sim 2$ ) with the ${ }^{18} \mathrm{O} /{ }^{16} \mathrm{O}$ isotopic ratio in the solar system. Figure c) shows similar calculations for ${ }^{22} \mathrm{Ne}$ assuming the $\mathrm{ACR}{ }^{22} \mathrm{Ne} /{ }^{20} \mathrm{Ne}$ ratio to be similar to (a) the GCR source, (b) meteorites and (c) solar wind. The data indicate an ACR Ne isotopic composition similar to the solar wind. 
Almost certainly, the Ne composition of the ACRs does not resemble that of GCRs (93, Figure 5c, option "a"), indicating that an additional source for ${ }^{22} \mathrm{Ne}$ other than just the ISM is required for GCRs. Better statistics could be reached with larger instruments and measurements in the outer heliosphere. Anomalous cosmic rays are also trapped and concentrated by a factor of 100 in the Earth's radiation belt. Measurements here could also be valuable $(94,95)$.

\section{Interstellar Probe Mission}

Figure 6 (84) illustrates the relative distribution of matter in the LISM in the three principal reservoirs, interstellar dust, neutral atoms, and plasma (data taken from 92). The fraction of material that is in the plasma state does not enter the heliosphere. Likewise, the majority of small interstellar grains are excluded from entry by the heliospheric magnetic field. Therefore, the most significant progress in sampling the properties of the interstellar medium would be gained by an interstellar probe mission $(84,96)$ that would provide direct access to these components of the LISM. The mission would allow an improved comparison of LISM and solar system composition, which should finally answer the question how closely the solar composition resembles that of the neighborhood in our Galaxy.

Furthermore, this mission could provide the measurement of cosmic rays outside of the heliosphere, inside which they experience energy loss. This would allow us to (possibly) determine the cosmic-ray spectrum in the ISM below $200 \mathrm{MeV} /$ nuc. Cosmic-ray induced spallation produces the light elements $\mathrm{Li}, \mathrm{Be}$, and B by three channels: a) GCR p and $\mathrm{He}$ interacting with ISM $\mathrm{C}$ and $\mathrm{O}$; b) GCR C, N, and $\mathrm{O}$ interacting with ISM $\mathrm{p}$ and $\mathrm{He}$; c) GCR He interacting with ISM He (Li only). Approximately, all of the $\mathrm{Li}, \mathrm{Be}$, and $\mathrm{B}$ produced via channel (a) by GCRs with energies of 200 to $2000 \mathrm{MeV} / \mathrm{nuc}$ is at thermal energies or will quickly thermalize. GCR $\mathrm{p}$ and $\mathrm{He}$ spectra at these energies are measured within the solar system. This channel contributes most of the spallogenic $\mathrm{Li}, \mathrm{Be}$, and $\mathrm{B}$. However, channel (b) contributes as well, although not as much as (a). Li, $\mathrm{Be}$, and $\mathrm{B}$ produced by channel (b) are almost as energetic as their GCR parents. Effectively all of the $\mathrm{Li}, \mathrm{Be}$, and $\mathrm{B}$ produced above $200 \mathrm{MeV} /$ nuc will escape before thermalizing. Therefore, channel (b) will only contribute if the GCR parents have energies of less than $200 \mathrm{MeV} / \mathrm{nuc}$. However, such a GCR spectrum cannot be measured inside the heliosphere because of solar modulation effects. The interstellar probe would thus help us to narrow down the GCR spallogenic contribution to $\mathrm{Li}, \mathrm{Be}$, and $\mathrm{B}$ galactic abundances at thermal energies.
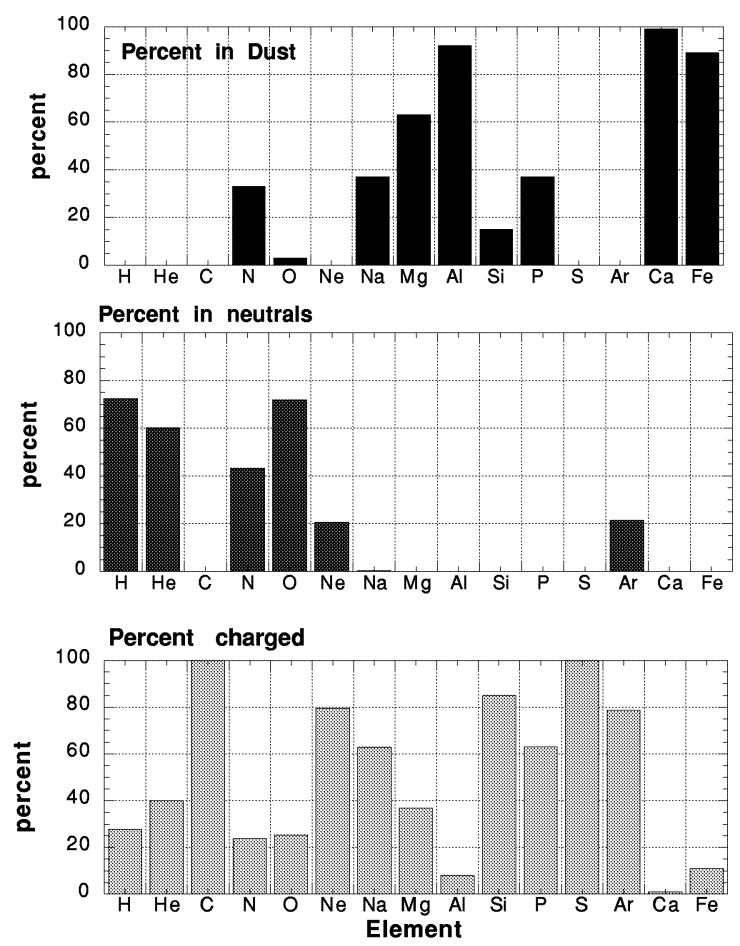

FIGURE 6. The distribution of the major elements in matter in the local interstellar medium as deduced theoretically (92). Figure reprinted from (84) with kind permission from Elsevier Science.

In addition to the investigations mentioned above, an Interstellar Probe mission would measure the magnetic field and plasma properties of the interstellar medium, investigate the interstellar spectrum of cosmic rays below $\sim 300 \mathrm{MeV} /$ nuc that are excluded from entering the heliosphere, and determine the source of the $2-3 \mathrm{kHz}$ radio emission that apparently originates near the nose of the heliosphere. During its passage through the outer solar system, it could survey the density of small (1 to $100 \mathrm{~km}$ ) Kuiper Belt objects from 30 to $200 \mathrm{AU}$ and thereby investigate the radial extent of the primordial solar nebula. This mission could also carry a small infrared telescope that would map the dust density in the outer solar system and measure the cosmic infrared background radiation in a wavelength region ( 5 to $100 \mu \mathrm{m}$ ) that is inaccessible to Earth-based telescopes because of obscuration by the zodiacal light (see 84 and 97 for additional information). 
In order to sample the material effectively throughout its journey, an "Interstellar Probe" must contain plasma, neutral gas, dust, and cosmic ray instruments with sufficient mass and charge resolution. This rather challenging mission is planned to reach a distance of $>200$ AU from the Sun within 15 years. The mission concept is currently based on solar sail propulsion with a gravity assist trajectory close to the Sun $(84,96)$. On its journey, the spacecraft would measure the elemental and isotopic composition of pickup ions, ACRs, neutrals, and dust in the outer heliosphere. Furthermore, the mission would examine - for the first time - the termination shock, the heliopause, possibly a bow shock beyond the heliosphere, and finally the local interstellar medium.

\section{GALACTIC COSMIC RAYS - WITNESSES OF STELLAR NUCLEOSYNTHESIS}

Nucleosynthesis processes and the resulting compositions of stars in the Galaxy cannot solely be studied spectroscopically. The galactic cosmic rays (GCRs) consist of particles that have been accelerated by shock waves in the vicinity of stars to energies up to several $\mathrm{GeV} /$ nuc. Subsequently, they propagate through the Galaxy and allow us to directly probe the average composition of the nucleosynthetic products of several stellar sources. A thorough understanding of this cosmic-ray propagation is important to identify how the cosmic-ray abundances are altered during transport and to determine the initial averaged composition of the cosmic-ray source. Here, we will discuss models that describe the physical propagation process and data needed to delimit the distinct possibilities. Another way to directly sample stellar compositions and decipher processes that lead to the production of nuclei in stars is the analysis of presolar or circumstellar grains that formed in the outflow of certain stars and survived the formation of the solar system unprocessed in primitive meteorites. This topic has been reviewed recently $(63,64)$ and is discussed by Hoppe in these proceedings (65, this volume). Therefore, we only discuss the importance of presolar grains for solar system abundances and galactic chemical evolution (see below).

\section{The Propagation of Galactic Cosmic Rays}

Isotopic and elemental abundance measurements of the galactic cosmic rays using instruments aboard balloons and spacecraft such as ACE, HEAO-3 (e.g., 98), Ulysses (e.g., 99), ISEE-3 (e.g., 100), IMP-7/8 (e.g., 101) and others, have provided insight into the nature of cosmic-ray propagation of particles with energies $\mathrm{E}_{\mathrm{ISM}}$ in the range of $200 \mathrm{MeV} /$ nuc to 50 $\mathrm{GeV} /$ nuc. The seed nuclei for galactic cosmic rays (GCRs) are synthesized in massive stars, supernovae, and possibly Wolf-Rayet (WR) stars. The GCR source nuclei experience a time delay of more than $10^{5} \mathrm{yrs}$ between their synthesis and their initial acceleration. This age was determined by observing the essentially complete decay of the e-capture isotope ${ }^{59} \mathrm{Ni}$ (102), which will decay only if the GCR source material remains at low energies where its electrons can remain attached for a period significantly longer than the ${ }^{59} \mathrm{Ni}$ half-life. Stable species such as the isotopes of boron, ${ }^{19} \mathrm{~F}$, and ${ }^{45} \mathrm{Sc}$ are produced predominantly via fragmentation during propagation (e.g., 103), and the abundances of these species indicate an average path length of $1-10 \mathrm{~g} / \mathrm{cm}^{2}$ in the energy range given above (e.g., 98). However, uncertainties in modeling GCR propagation remain that can be overcome by new measurements.

Is GCR propagation described accurately by a "leaky-box" model or are more realistic diffusion models required? The leaky-box model assumes that all GCRs freely diffuse within a confinement region (or "box") containing an approximately homogeneous interstellar medium (ISM) matter density, with a finite probability for escape at the boundary. However, more realistic diffusion models suggest that one cannot treat the ISM as homogeneous, and that the average value of the ISM density probed by GCRs depends on the size of the propagation volume. One can test this dependence by measuring abundances of GCR species that are produced only via fragmentation during propagation and decay via $\beta$-emission. The abundances of these GCR "clocks" will depend on the time of cosmic-ray confinement in the Galaxy and the rate of fragmentation in the ISM (and in turn the ISM density in the propagation volume probed by each species). The surviving fractional abundances of these secondary $\beta$-decay radionuclides (e.g. ${ }^{10} \mathrm{Be},{ }^{26} \mathrm{Al},{ }^{36} \mathrm{Cl}$, ${ }^{54} \mathrm{Mn}$ ) measured at $\mathrm{E}_{\mathrm{ISM}}=200-500 \mathrm{MeV} /$ nuc imply a propagation time $\tau_{\text {esc }}$ of $15 \mathrm{Myr}$ and an average ISM hydrogen density $\rho_{\text {ISM }}$ of $0.34 \mathrm{H}$ atoms $/ \mathrm{cm}^{3}$ (104). All of the individual $\tau_{\text {esc }}$ and $\rho_{\text {ISM }}$ values from measurements of these species are consistent within measured uncertainties with a unique value of $\rho_{\text {ISM }}$ and $\tau_{\text {esc }}$ as assumed by the leaky-box model. However, the decay time of the GCR clock species will experience relativistic time dilatation at higher energies, so the propagation volume is correspondingly larger. 


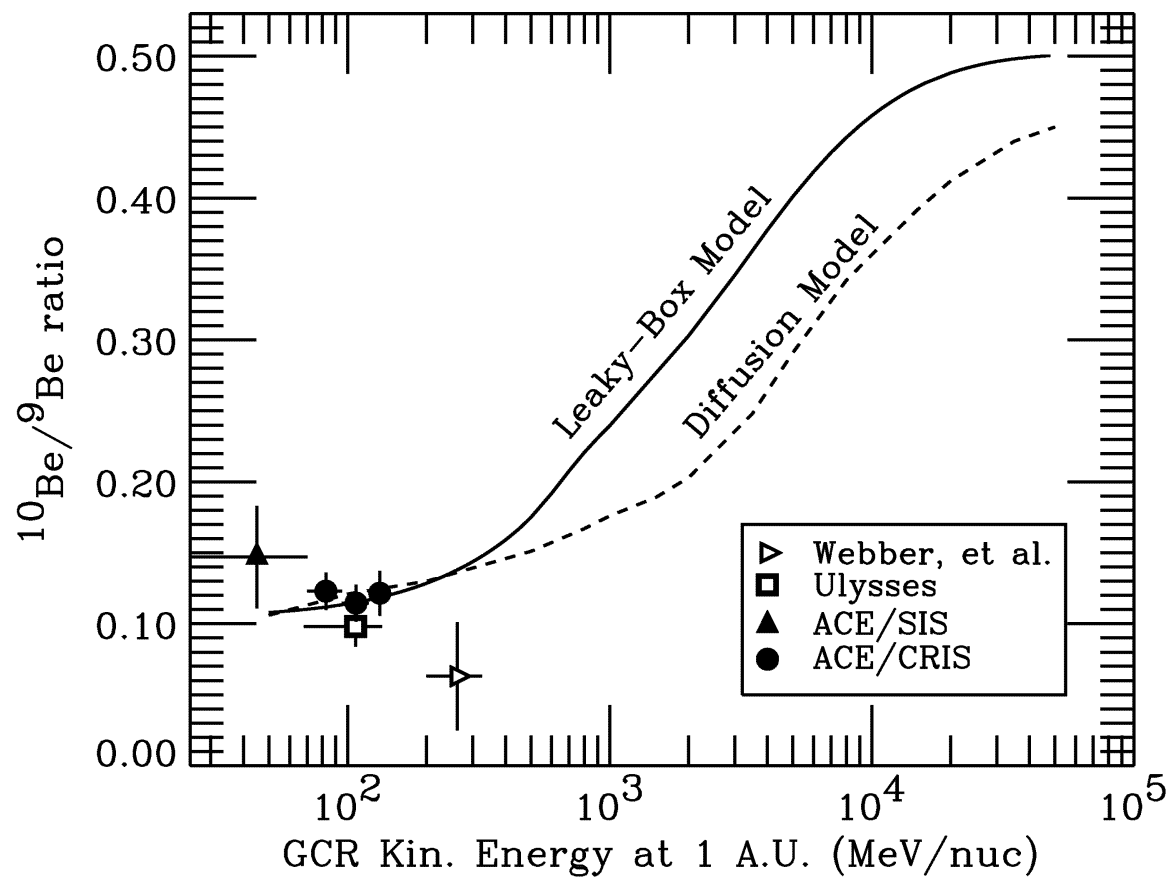

FIGURE 7. ${ }^{10} \mathrm{Be} /{ }^{9} \mathrm{Be}$ ratios as measured in the GCR at lower energies $(104,105,108)$. Data points obtained for GCRs at energies in the range $1-10 \mathrm{GeV} /$ nuc with sufficiently small uncertainties could distinguish between the models that describe the propagation of GCRs.

The inhomogeneity predicted in diffusion models leads to a decrease in the average ISM density $\rho_{\text {ISM }}$ with increasing propagation volume. Thus, abundance measurements at both low and high energies will help distinguish between the two models (Figure 7).

Figure 7 shows low-energy GCR data measured by the ACE/CRIS and ACE/SIS instruments (104) and Ulysses (105) for the ${ }^{10} \mathrm{Be} /{ }^{9} \mathrm{Be}$ ratio. Also shown are ${ }^{10} \mathrm{Be} /{ }^{9} \mathrm{Be}$ ratio curves calculated from a leaky-box model (106) for a density of $\rho_{\text {ISM }}=0.34 \mathrm{H}$ atoms $/ \mathrm{cm}^{3}$ and a diffusion model (107) for a galactic halo size of $\mathrm{z}(\mathrm{h})=2 \mathrm{kpc}$. Measurements of ${ }^{10} \mathrm{Be} /{ }^{9} \mathrm{Be}$ at higher energies have been performed by the balloon experiments of Webber and Kish (108), SMILI (109), and ISOMAX $(110,111)$. The recent ISOMAX results, which extend from $\sim 0.3$ to $2 \mathrm{GeV} /$ nuc, give values of $\sim 0.2$ at $0.65 \mathrm{GeV} /$ nuc and $\sim 0.3$ at $\sim 1.5$ $\mathrm{GeV} /$ nuc, consistently above the diffusion model in Figure 7. However, given the uncertainties, it is not yet possible to distinguish between the leaky-box model and a diffusion model, possibly characterized by a somewhat different halo size and/or diffusion coefficient. Measurements of the ${ }^{10} \mathrm{Be} /{ }^{9} \mathrm{Be}$ ratio (see figure 7) at two higher energies between 1-10 $\mathrm{GeV} / \mathrm{nuc}$ to $11 \%$ uncertainty (systematic and statistical) or four energies to $15 \%$ uncertainty could distinguish between the competing models discussed above to within $3 \sigma$, or could motivate a new physical resolution to this problem. In addition, high-energy measurements of the ${ }^{26} \mathrm{Al} /{ }^{27} \mathrm{Al}$ ratio to a similar accuracy could identify whether a density variation probed by a particular species is the same for other clocks.

\section{The Abundances of Cosmic Ray Nuclei beyond the Iron Peak}

In nature, nuclei with $31 \leq Z \leq 92$ are extremely rare. Their abundances amount in total to only $\sim 3 \times 10^{-4}$ of the Fe abundance. In cosmic rays, nuclei with $\mathrm{Z} \geq$ 30 are of particular interest because they can address two important issues, the nature of the material that is accelerated to be cosmic rays and the age of the material since it was originally synthesized.

Most of the nuclei with $Z \geq 30$ are produced by neutron-capture processes that can be modeled in terms of two extremes - a slow-process (s-process) that permits $\beta$-decay before additional neutron captures, and a rapid process (r-process), resulting in multiple neutron captures before $\beta$-decay. Previous space experiments (see, e.g., 112 and references 
therein) observed an underabundance of nuclei in the "Pb-group" ( $81 \leq \mathrm{Z} \leq 83)$ relative to those in the "Ptgroup" ( $74 \leq Z \leq 80)$, and interpreted this as due to an overabundance of r-process nuclei in cosmic rays relative to the r-process/s-process mix of material in the solar system. However, Westphal et al. (113), who confirmed that the $\mathrm{Pt} / \mathrm{Pb}$ ratio is enhanced in cosmic rays, suggested that this was due to atomic fractionation effects associated with the formation of dust grains, which may be the source of the refractory elements in cosmic rays (114).

It is well known that cosmic rays contain an overabundance of nuclei with first ionization potential (FIP) $<10 \mathrm{eV}$, relative to those with FIP $>10 \mathrm{eV}$. A similar pattern is observed in solar energetic particles and in the solar wind, indicating that the corona is enhanced in elements with FIP $<10 \mathrm{eV}$ by about a factor of $\sim 3$ to 4 . One possible explanation for the occurrence of the FIP effect in cosmic rays is that they may originate as nuclei accelerated in stellar flares of Sun-like stars, which are then accelerated to higher energies by supernova shocks (115). However, Meyer, Drury, and Ellison (114) argued that the apparent correlation with FIP is coincidental, and that it is actually the volatility of the elements that is important. They suggested that cosmic rays originate from interstellar dust grains that are accelerated to energies of $\sim 0.1$ to $1 \mathrm{MeV} /$ nuc. Ions sputtered from these grains (mostly refractory species) can then be efficiently accelerated to cosmic ray energies along with an appropriate mixture of interstellar gas (mostly volatile elements).

Although the atomic properties FIP and volatility are highly correlated, there are a number of elements in with $30 \leq \mathrm{Z} \leq 83$ (e.g., ${ }^{31} \mathrm{Ga},{ }^{32} \mathrm{Ge},{ }^{34} \mathrm{Se}$, ${ }^{37} \mathrm{Rb},{ }^{47} \mathrm{Ag},{ }^{50} \mathrm{Sn},{ }^{55} \mathrm{Cs}$ and $\left.{ }^{82} \mathrm{~Pb}\right)$ that can distinguish between these two models (114), if one could measure the abundances of individual cosmic rays species with sufficient accuracy. The proposed Heavy Nuclei Explorer (HNX) mission, now under study by NASA, will measure the abundances of cosmic rays with $10 \leq$ $\mathrm{Z} \leq 96$ with $\sim 10$ times the collecting power of previous experiments, and with individual element resolution (116). HNX consists of the ENTICE instrument, which measures nuclei with $10 \leq Z \leq 83$, and the ECCO instrument, which uses glass track-etch detectors to measure nuclei with $\mathrm{Z}>70$.

In addition to answering the question as to whether cosmic rays originate as grain-destruction products, HNX would also collect anywhere from $\sim 100$ to $\sim 300$ actinide nuclei (those with $90 \leq Z \leq 96$ ), sufficient to measure the age of cosmic ray nuclei since their nucleosynthesis. If cosmic rays are accelerated from the ISM, the mean age will be several Gyrs, and at least 100 actinides would be expected in a 2-year mission. If cosmic rays are enhanced in r-process nuclei, a large fraction of these nuclei must be very young, permitting short-lived elements such as ${ }^{96} \mathrm{Cm}$ to survive. In the case where cosmic rays originate in newly synthesized material, 300 actinides would be expected. In the model of Higdon et al. (117), in which cosmic rays originate in superbubbles, there is predicted to be a dramatic enhancement in freshlysynthesized r-process material, with an age of $\sim 10^{7}$ yrs, characteristic of an $\mathrm{OB}$ association. In addition to addressing the important issues of FIP vs. volatility, and the cosmic rays age since nucleosynthesis, HNX would determine the r-process and s-process mix of material among elements with $30 \leq \mathrm{Z} \leq 83$.

\section{GALACTIC ABUNDANCES}

For this contribution, we have chosen situations, where measured solar or meteoritic and ISM abundance ratios can be used to set tight constraints both to chemical evolution and stellar evolution models.

\section{Galactic Chemical Evolution and Presolar Grains}

It has been argued by Clayton (118) that presolar dust found in meteorites preserves a memory of the evolution of the abundances of the chemical elements in the Galaxy with time, the galactic chemical evolution (GCE). In fact, the distribution of Si-isotopic ratios measured in presolar $\mathrm{SiC}$ grains separated from primitive meteorites (66) cannot be reconciled with evolutionary models of the parent stars (AGB stars, or more specifically carbon stars) but may reflect the GCE of the Si isotopes, both in time and space (119, 120 , see also 65 , this volume). As the stardust in meteorites formed at different times and locations in our Galaxy, its isotopic compositions can be used to test models of GCE. In general, the isotopic compositions of stardust are determined by the starting compositions of the parent stars (reflecting the GCE) and the nucleosynthesis and evolution of these stars during their lifetime. The GCE is best recognized if the effects of stellar nucleosynthesis and evolution on the isotopic compositions at the parent star's surface are small compared to the spread of isotopic compositions at the times the parent stars formed. 


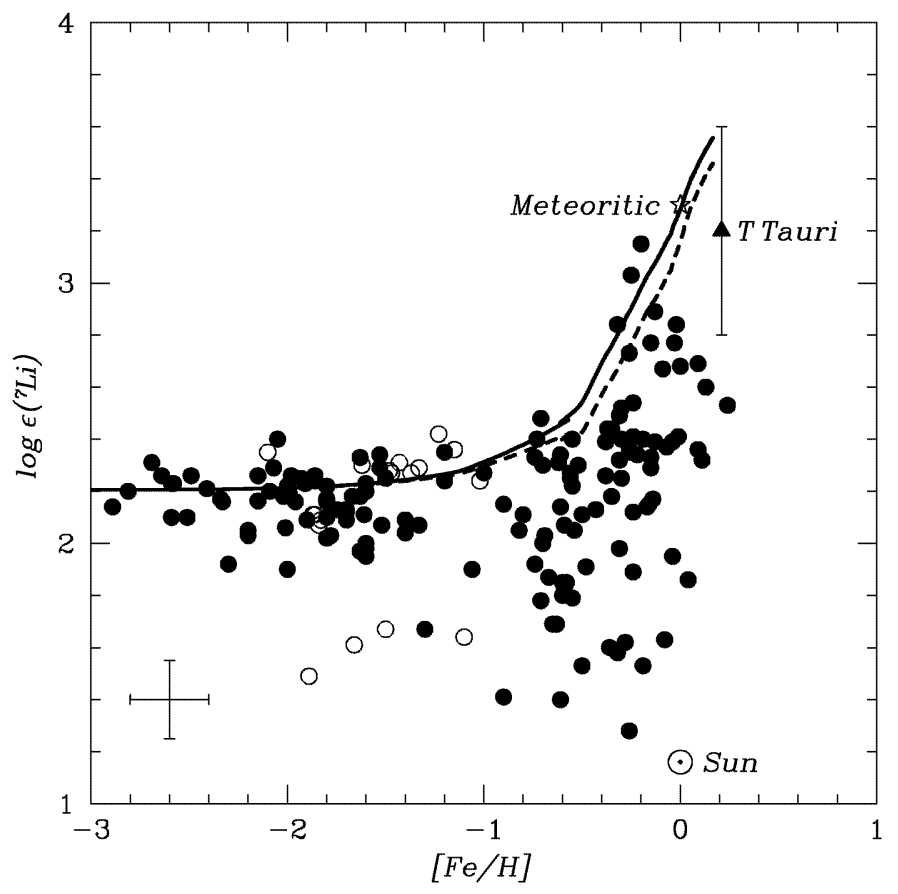

FIGURE 8. $\log \varepsilon\left({ }^{7} \mathrm{Li}\right)\left(\equiv " \mathrm{~A}(\mathrm{Li}) " \equiv \log \left(\mathrm{N}_{7 \mathrm{~L} i} / \mathrm{N}_{\mathrm{H}}\right)+12.0\right)$ vs. $[\mathrm{Fe} / \mathrm{H}]\left(\equiv \log \left(\mathrm{N}_{\mathrm{Fe}} / \mathrm{N}_{\mathrm{H}}\right)-\log \left(\mathrm{N}_{\mathrm{Fe}} / \mathrm{N}_{\mathrm{H}}\right)_{\odot}\right)$ theoretical trends predicted by models of Romano et al. (124, figure reprinted with kind permission from EDP Sciences) including all astrophysical sites for ${ }^{7} \mathrm{Li}$ production (Data from 121, filled circles, and 149, open circles). Dashed line: Type II SNe + AGB stars + low-mass giants + novae. Continuous line: Type II SNe + AGB stars + low-mass giants + novae + GCRs (their best model).

Besides $\mathrm{Si}$ and $\mathrm{Ti}$, elements of interest include $\mathrm{Mg}$ in spinel (and silicates) and $\mathrm{Ca}$ and $\mathrm{Ti}$ in hibonite and other $\mathrm{Ca}$ - or Ti-bearing minerals from red giant and AGB stars. As the expected isotopic effects due to GCE are on the order of $10 \%$ (cf. Si in SiC), isotopic compositions of individual presolar grains should be measured with accuracy on the order of a few $\%$.

Contemporary interstellar dust will be collected by the STARDUST mission and brought to Earth in the year 2006 (19). A comparison of its isotopic compositions with those of presolar grains from meteorites that formed more than 4.6 Gyrs ago will allow to obtain additional information on the GCE of certain elements.

\section{Evolution of Lithium in the Milky Way}

To explain the ${ }^{7} \mathrm{Li}$ evolution in the Galaxy, several sites of production and destruction of this isotope have to be considered, namely Big Bang nucleosynthesis (BBN), spallation reactions between GCRs and the ISM, and stellar nucleosynthesis (121). In particular, the measurement of the ${ }^{7} \mathrm{Li}$ abundance in distinct stellar objects of different populations and its temporal variation as predicted by chemical evolution models lead to an estimate of the primordial abundance of ${ }^{7} \mathrm{Li}$. This abundance as well as those of $\mathrm{D},{ }^{3} \mathrm{He}$, and ${ }^{4} \mathrm{He}$, produced during the Big Bang, are of fundamental importance to probe the consistency of the BBN theory and provide a valuable constraint on the baryon density of the universe (122).

The ${ }^{7} \mathrm{Li}$ abundances as observed in the solar neighborhood are shown in Figure 8. The upper envelope of the data, as plotted in the $\log \varepsilon\left({ }^{7} \mathrm{Li}\right)$ vs. $[\mathrm{Fe} / \mathrm{H}]$ diagram, is generally believed to reflect the ${ }^{7} \mathrm{Li}$ enrichment history of the LISM in the solar neighborhood. Therefore, it can be used to constrain models of galactic chemical evolution. The envelope is characterized by i) a large plateau at low metallicity, the so-called Spite plateau (123) and ii) a steep rise afterwards (124 and references therein) due to the ${ }^{7} \mathrm{Li}$ production by stellar nucleosynthesis and spallation of cosmic rays.

Data points below the envelope indicate that $\mathrm{Li}$ was depleted due to mixing of the surface layers of the respective stars with their interiors (convection). The efficiency of this mixing depends essentially on the stellar temperature (125). The solar photosphere 
contains less than $1 \%$ of the Li present in the ISM at the time of formation of the Sun (Figure 8), which is well represented by $\mathrm{Li}$ in meteorites $(1, \mathrm{cf}$. Figure 1$)$.

Generally, ${ }^{7} \mathrm{Li}$ in warm halo population II stars with very low metallicity (Spite plateau) is considered to represent its primordial abundance $(122,126)$. Some depletion of ${ }^{7} \mathrm{Li}$ in pop II stars might be possible, but the low dispersion of the lithium data at the Spite plateau suggests that this depletion cannot be large. One way to address this issue is to compare the primordial ${ }^{7} \mathrm{Li}$ abundance, as estimated by using the deuterium-inferred value of the baryon density, with the Spite plateau value (127).

Adopting up-to-date stellar yields for Novae, AGB stars, Type II SNe and Carbon stars, Romano et al. (124) conclude that: i) Type II SNe and novae are necessary in order to reproduce the ${ }^{7} \mathrm{Li}$ abundance evolution represented by the upper envelope of the observational data; ii) when adopting the new stellar ${ }^{7} \mathrm{Li}$ for AGB stars, those stars can no longer be considered as a significant source of ${ }^{7} \mathrm{Li}$ in the Galaxy; iii) Novae (and probably low-mass giant stars) restoring their processed material on long timescales, are among the best candidates for reproducing the late rise from the Spite plateau.

\section{The D, ${ }^{3} \mathrm{He}$, and ${ }^{4} \mathrm{He}$ Galactic Evolution and their Abundance Gradient}

Chemical evolution models are useful to derive both the primordial abundances of $\mathrm{D},{ }^{3} \mathrm{He}$, and ${ }^{4} \mathrm{He}$ as well as their evolution. In the light of recent observations, Chiappini et al. developed a new model for the Galaxy, the so-called two-infall model (128, 129 , this volume). Predictions of this model for the chemical evolution of $\mathrm{D},{ }^{3} \mathrm{He}$, and ${ }^{4} \mathrm{He}$ in the solar vicinity and for their distribution along the galactic disk are described in the following.

The model assumes two main gas infall episodes for the formation of the halo (and part of the thick disk) and thin disk, respectively (see 128 for references). The timescale for the formation of the thin disk is much longer than that of the halo, implying that the infalling gas forming the thin disk not only comes from the halo but mainly from the intergalactic medium. The formation time of the thin disk is assumed to be a function of galactocentric distance, leading to an "inside-out" picture for the Galaxy disk buildup, where the inner part accreted much faster than the outer regions (130). Figure 9 shows the predictions of the two-infall model for the evolution of ${ }^{3} \mathrm{He}$ and $\mathrm{D}$ in the solar vicinity. The figure indicates a solution for a long-standing problem in chemical evolution, namely the overestimation of ${ }^{3} \mathrm{He}$ by the models compared to the values observed in the Sun and the interstellar medium. The solution (129, this volume) requires allowing for "extra-mixing" in low-mass stars $\left(\mathrm{M}<2.5 \mathrm{M}_{\odot}, 131\right)$. This "non-standard" extra-mixing occurs in RGB stars between the bottom of the convective envelope and the H-burning shell (131).

Chemical evolution models can also constrain the primordial value of the deuterium abundance. The primordial abundances by mass of $\mathrm{D}$ and ${ }^{3} \mathrm{He}$ were taken for the calculations to be $4.4 \times 10^{-5}$ and $2.0 \times$ $10^{-5}$, respectively. While this $\mathrm{D}$ primordial value represents an upper limit (see Figure 9a), the adopted ${ }^{3} \mathrm{He}$ abundance must be a lower limit (Figure 9b). The present model (129, this volume) suggests a value of $(\mathrm{D} / \mathrm{H})_{\text {prim }}<3 \times 10^{-5}$ (by number). This is in agreement with the low primordial $\mathrm{D} / \mathrm{H}$ value of $(3.0 \pm 0.4) \times 10^{-5}$ deduced from the Ly $\alpha$ feature in spectra of four highly redshifted $(\mathrm{z}>3)$ low metallicity quasar absorption systems (QAS) (132, 133 and references therein). Other measurements indicating a significantly higher $\mathrm{D} / \mathrm{H}$ may be explained with $\mathrm{H}$ contamination (132).

The primordial $\mathrm{D}$ abundance is considered to be the best baryometer, because it strongly depends on the baryon density. Observed abundances of D are lower limits on $\mathrm{D}_{\text {prim }}$ and thus upper limits of the baryon density, because D is only destroyed in post-BBN astrophysical processes. This ratio thus probes the consistency of BBN and constrains D chemical evolution. Vice versa, the low primordial D value from QAS may serve as a test for chemical evolution modeling. Observations of the LISM (134) and the solar system (135) represent tight constraints to the evolution of the D primordial abundance (Figure 9a; see 136 for possible $\mathrm{D}$ abundance variations in the LISM). Models that can reproduce the bulk of the observational data predict only a modest $\mathrm{D}$ destruction (in our case a factor <1.6; see 137).

The predicted radial abundance gradient for $\mathrm{D}$ (see 138 ) is positive and steep, due to the faster evolution of the inner disk regions compared with the outer parts (which are still in the process of formation and thus having an almost primordial composition). Of the various elements, $\mathrm{D}$ is probably most sensitive to radial variations in the timescale of disk formation. Thus, precise D abundance measurements in regions outside the solar vicinity are needed. 

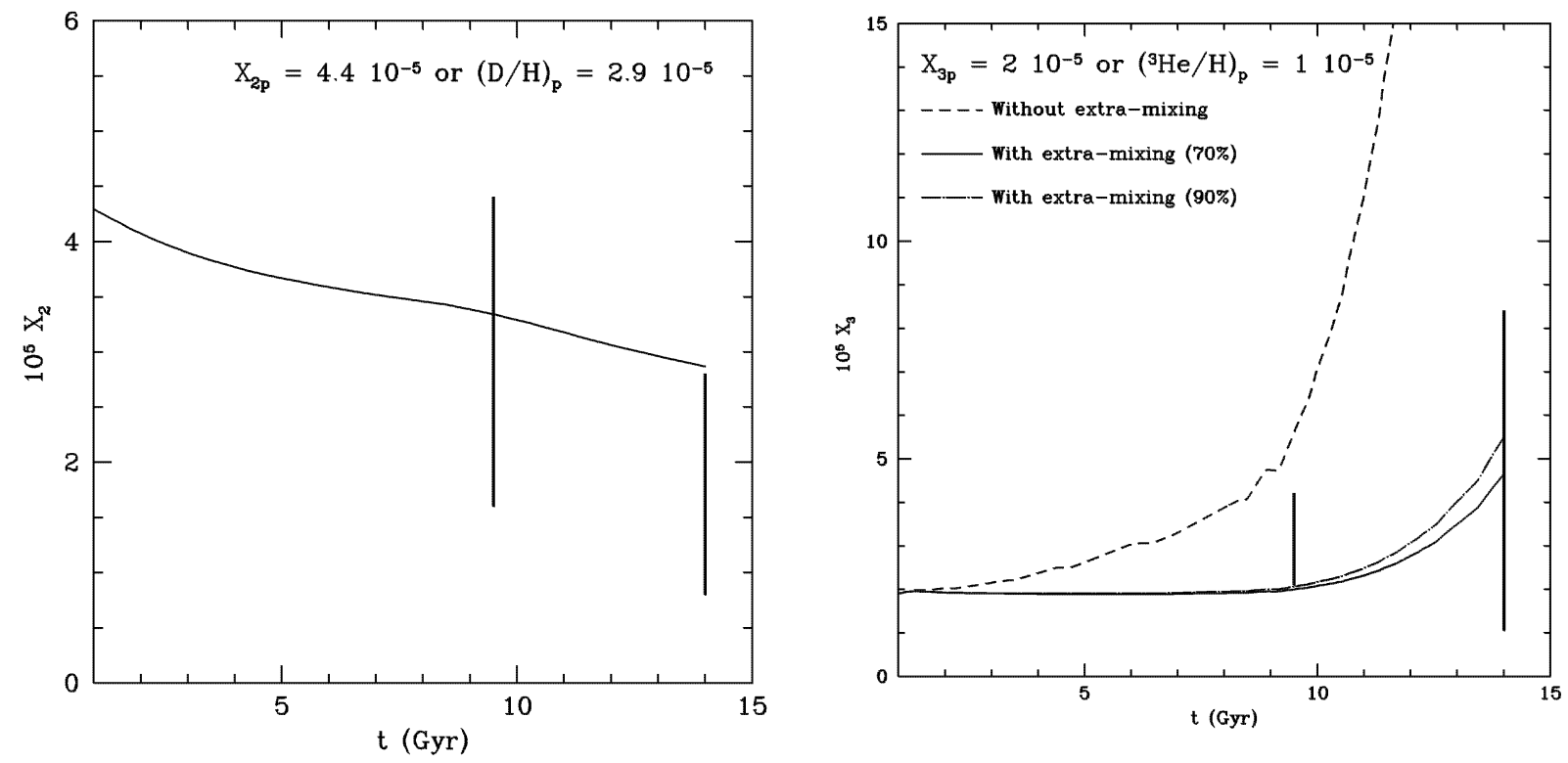

FIGURE 9. a) D and b) ${ }^{3} \mathrm{He}$ evolution (by mass) as predicted in 138 , reprinted with kind permission from the Astronomical Society of the Pacific. The bars at 9.5 Gyr (4.5 Gyrs ago) and 14 Gyr (age of the Galaxy) represent the value of solar (2 $\sigma$, 135) and $\operatorname{ISM}(2 \sigma, 134)$ abundances, respectively.

For the ${ }^{3} \mathrm{He}$ abundance gradient (138), the assumption that a high fraction of low-mass stars suffers extra mixing leads to a flat gradient along the disk, which is in quite good agreement with the observed gradient measured in HI regions (139). Models without extra-mixing predict too much ${ }^{3} \mathrm{He}$ in the last Gyrs of the evolution of the Galaxy (Figure $9 b$ ). In this case, the ${ }^{3} \mathrm{He}$ abundance gradient would be sensitive to the adopted timescales of disk formation. Without extra-mixing and subsequent ${ }^{3} \mathrm{He}$ destruction in low-mass stars, we would predict that in the inner regions (older in the inside-out scenario) the contribution of these stars for the ${ }^{3} \mathrm{He}$ enrichment of the ISM would be more important than in the outer regions, and therefore a negative gradient would have been formed towards the outer regions of the disk. More data on the ${ }^{3} \mathrm{He}$ abundance at different galactocentric distances are thus very important to better constrain the chemical evolution models. These data as well constrain models of low-mass stellar nucleosynthesis.

Finally, adopting the primordial ${ }^{4} \mathrm{He}$ abundance suggested in (140), the model (138) yields a value for the galactic enrichment of $\mathrm{He}$ relative to the heavy elements $\Delta Y / \Delta Z \cong 2$ and a better agreement with the solar ${ }^{4} \mathrm{He}$ abundance (see summary of results and figures in 138). The ${ }^{4} \mathrm{He}$ gradient with galactocentric distance is rather flat $(\cong-0.003 \mathrm{dex} / \mathrm{kpc}$ over the $4-14$ $\mathrm{kpc}$ galactocentric range) in agreement with the results on disk planetary nebulae (141). These results should be used with caution, because at present, the primordial ${ }^{4} \mathrm{He}$ abundance, $\mathrm{Y}_{\mathrm{p}}$, inferred from observations still suffers from systematic errors $\left(\mathrm{Y}_{\mathrm{p}}=\right.$ $0.228 \pm 0.005$ vs. $0.244 \pm 0.002,142,143)$. Future measurements of helium with a decreased systematic error will allow using the ${ }^{4} \mathrm{He}$ abundance as a precision test for $\mathrm{BBN}$, standard cosmology, and chemical evolution modeling. At present, a theoretically calculated $\mathrm{Y}_{\mathrm{p}}$ value is usually used to constrain physics beyond the standard model (144, this volume, 145 and references therein).

It is interesting to notice that chemical evolution models can be used to constraint models of big bang nucleosynthesis (e.g., 144) as far as the primordial abundances of the light elements are inferred from observational data, accounting for their corresponding chemical and stellar evolution. On the other hand, standard $\mathrm{BBN}$ is a powerful tool for constraining models of chemical evolution, non-standard cosmological models and physics beyond the standard model, because the four light element abundances are predicted based on only one parameter - the baryon density. Hence, new observational data of the light elements $\mathrm{D},{ }^{3} \mathrm{He},{ }^{4} \mathrm{He}$, and ${ }^{7} \mathrm{Li}$ are essential. 

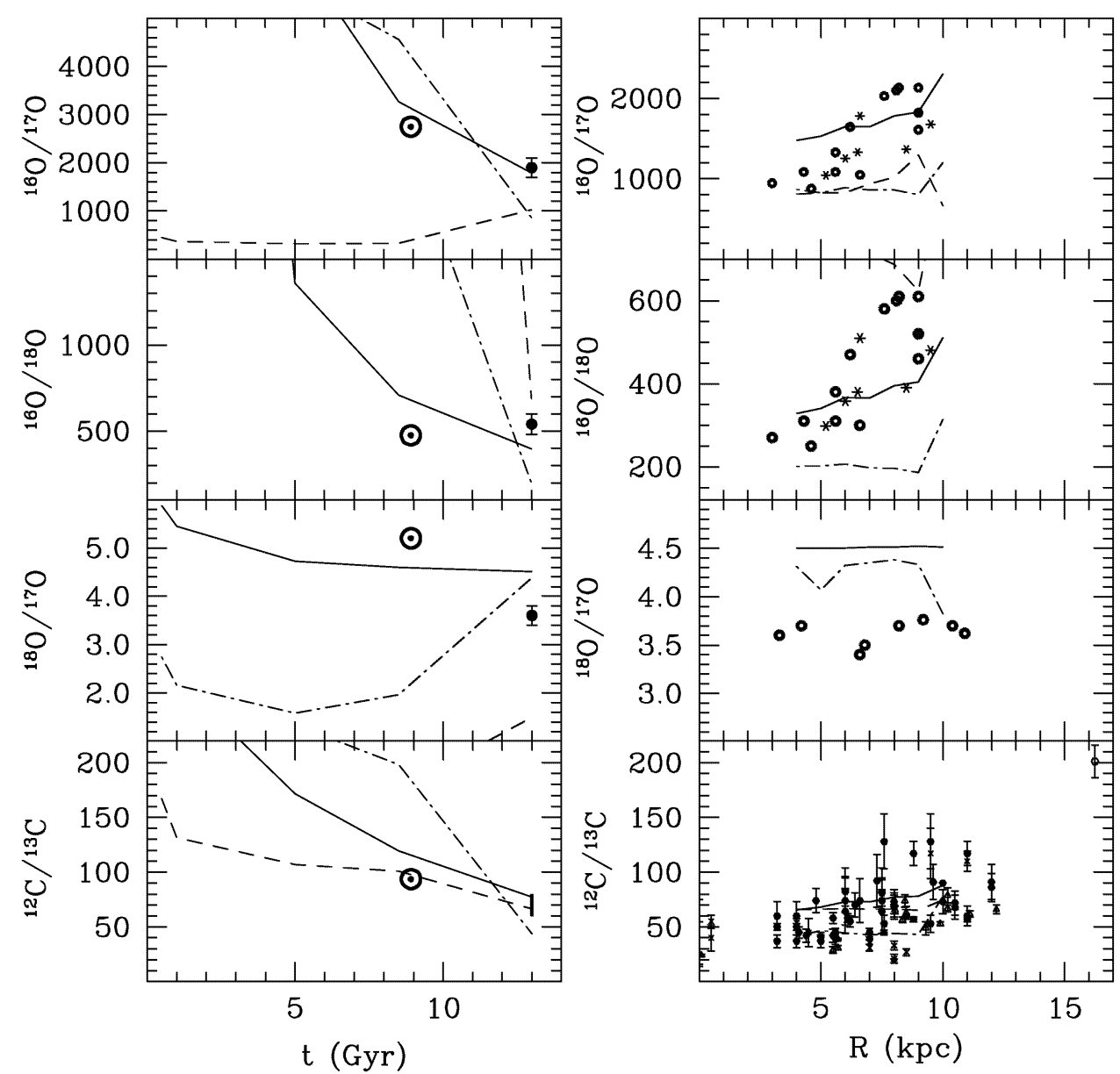

FIGURE 10. Carbon (bottom panels) and oxygen (three top panels) isotopic ratios. Left panels: evolution in the solar neighborhood. Right panels: current distributions with galactocentric distance. The solar symbol represents the solar ratio derived from Anders \& Grevesse (1); all the other data are from radio observations of molecular clouds (146 and references therein). Models and figure are from Tosi (146, reprinted with kind permission from Kluwer Academic Publishers).

\section{Abundance Ratios as a Function of Metallicity}

Abundance ratios of a primary element (its production is independent of the initial heavy element abundance of the star) over a secondary one (its precursor element is produced in previous generation stars and its abundance is proportional to the initial heavy element abundance) are expected to decrease with time or metallicity (125). This can be useful to understand the origin of different elements and to give us information on the timescale of formation of a certain system such as the solar vicinity. Moreover, the abundance ratio of two primary elements that are restored to the interstellar medium by stars in different mass ranges would show almost the same behavior discussed above. One example is the ${ }^{16} \mathrm{O} /{ }^{12} \mathrm{C}$ ratio. Both elements are primary but since ${ }^{12} \mathrm{C}$ is mainly restored into the interstellar medium by intermediate mass stars (and hence on large timescales compared to the ${ }^{16} \mathrm{O}$ enrichment that comes mainly from massive stars), this ratio decreases as a function of metallicity.

The isotopic ratios of $\mathrm{C}, \mathrm{N}$, and $\mathrm{O}$ are of particular interest (Figure 10). As discussed recently by Tosi (146), chemical evolution models have difficulties in explaining the behavior of some of the isotopic ratios. The solar and ISM values for ${ }^{13} \mathrm{C} /{ }^{12} \mathrm{C},{ }^{17} \mathrm{O} /{ }^{16} \mathrm{O},{ }^{18} \mathrm{O} /{ }^{16} \mathrm{O}$ and ${ }^{15} \mathrm{~N} /{ }^{14} \mathrm{~N}$, as well as their radial profiles in the Galaxy, are very important as they can be used to 
constrain the mechanisms of disk formation and particular stellar evolution models.

For example, the steep rise of the ${ }^{13} \mathrm{C} /{ }^{12} \mathrm{C}$ with metallicity -or time- can be explained by novae contributions to ${ }^{13} \mathrm{C}$ or by the extra-mixing mechanism discussed above, which consumes ${ }^{3} \mathrm{He}$ and produces ${ }^{13} \mathrm{C}$ in low-mass stars $(147,148)$. The oxygen isotopes need further examination because the models (146) predict an increase of the ${ }^{18} \mathrm{O} /{ }^{17} \mathrm{O}$ ratio from the solar to the local ISM value and a corresponding decrease of ${ }^{16} \mathrm{O} /{ }^{18} \mathrm{O}$, contrary to what is observed (see 146 for references). The predictions involving ${ }^{18} \mathrm{O}$ are not in agreement with the observations, whereas chemical evolution models can well explain the observed behavior of ${ }^{12} \mathrm{C} /{ }^{13} \mathrm{C}$ and ${ }^{16} \mathrm{O} /{ }^{17} \mathrm{O}$. The discrepancies in ${ }^{18} \mathrm{O}$ seem to be related to stellar evolution computations, because the observed values for ${ }^{18} \mathrm{O}$ (at least that in the Sun) are, for this purpose, sufficiently well known. The increase of some of the ratios mentioned above as a function of galactocentric distance (Figure 10) could be understood based on the "inside-out" picture for galactic disk formation (129, this volume). At present, the inner parts of the Galaxy are more evolved and thus present a larger abundance of secondary elements compared with the outer regions.

\section{ACKNOWLEDGMENTS}

We thank the organizing committee of the SOHO/ACE Workshop held at the University of Bern, Switzerland, for bringing us together. This resulted in fruitful cross-disciplinary discussions, which we hope to be at least partly reflected in this contribution.

\section{REFERENCES}

1. Anders, E., and Grevesse, N., Geochim. Cosmochim. Acta 53, 197-214 (1989).

2. Palme, H., and Beer, H., "Abundances of the elements in the solar system" in Landolt Börnstein Vol. VI/3a, New Series, Astronomy and Astrophysics, edited by H. H. Voigt, Berlin: Springer, 1993, pp. 196-221.

3. Cunha K., and Smith, V. V., Astrophys. J. 512, 10061013 (1999).

4. Begemann, F., Rep. Progr. Phys. 43, 1309-1356 (1980).
5. Huss, G. R., and Alexander Jr., E. C., Proc. 7th Lunar Planet. Sci. Conf., J. Geophys. Res. 92, E710-E716 (1987).

6. Porcelli, D., and Pepin, R. O., "Rare gas constraints on early earth history" in Origin of the Earth and Moon, edited by R. M. Canup and K. Righter, Tucson: University of Arizona Press, 2000, pp. 435-458.

7. Owen, T., Mahaffy, P., Niemann, H. B., Atreya S., Donahue, T., Bar-Nun, A., and de Pater, I., Nature 402, 269-270 (1999).

8. Christensen-Dalsgaard, J., Space Sci. Rev. 85, 19-36 (1998).

9. Bochsler, P., Rev. Geophys. 38, 247-266 (2000).

10. Feldman, U., Space Sci. Rev. 85, 227-240 (1998).

11. Wieler, R., Space Sci. Rev. 85, 303-314 (1998).

12. Pepin, R. O., Becker, R. H., and Rider, P. E., Geochim. Cosmochim. Acta 59, 4997-5022 (1995).

13. Geiss, J., Bühler, F., Cerutti, H., Eberhardt, P., and Filleux, C., Apollo 16 Prelim. Sci. Rep. NASA SP-315, 14.1-14.10 (1972).

14. Bodmer, R., and Bochsler, P., Astron. Astrophys. 337, 921-927 (1998).

15. Kallenbach, R., Ipavich, F. M., Kucharek, H., Bochsler, P., Galvin, A. B., Geiss, J., Gliem, F., Gloeckler, G., Grünwaldt, H., Hefti, S., Hilchenbach, M., and Hovestadt, D. Space Sci. Rev. 85, 357-370 (1998).

16. Weygand, J. M., Ipavich, F. M., Wurz, P., Paquette, J. A., and Bochsler, P., Geochim. Cosmochim. Acta, in press (2001).

17. Chaussidon, M., and Robert, F., Nature 402, 270-273 (1999).

18. McKeegan, K. D., Chaussidon, M., and Robert, F., Science 289, 1334-1337 (2000).

19. Huntress Jr., W. T., Space Sci. Rev. 90, 329-340 (1999).

20. Altwegg, K., Balsiger, H., and Geiss, J., Space Sci. Rev. 90, 3-18 (1999).

21. Feldman, U., and Laming, J. M., Physica Scripta 61, 222-252 (2000).

22. Boothroyd, A. I., Sackmann, I.-J., and Fowler, W. A., Astrophys. J. 377, 318-329 (1991).

23. Wiens, R. C., Burnett, D. S., Neugebauer, M., and Pepin, R. O., Proc. 22th Lunar Planet. Sci. Conf., 153-159 (1992). 
24. Clayton, R. N., Annual Rev. Earth Planet. Sci. 21, 115149 (1993).

25. Collier, M. R., Hamilton, D. C., Gloeckler, G., Ho, G., Bochsler, P., Bodmer, R., and Sheldon, R., J. Geophys. Res. 103, 7-13 (1998).

26. Wimmer-Schweingruber, R. F., Bochsler, P., and Gloeckler, G., Geophys. Res. Lett. 28, 2763-2766 (2001).

27. Leske, R. A., Mewaldt, R. A., Cohen, C. M. S., Christian, E. R., Cummings, A. C., Slocum, P. L., Stone, E. C., von Rosenvinge, T. T., and Wiedenbeck, M. E., AIP Conf. Proc. this volume (2001).

28. Eberhardt, P., Reber, M., Krankowsky, D., and Hodges, R. R., Astron. Astrophys. 302, 301-316 (1995).

29. Thiemens, M. R., and Heidenreich, J. E., Science 219, 1073-1075 (1983).

30. Wiens, R. C., Huss, G. R., and Burnett, D. S., Meteorit. \& Planet. Sci. 34, 99-107 (1999).

31. Kerridge, J. F., Science 188, 162-164 (1975).

32. Clayton, R. N., and Thiemens, M. R., Proc. Conf. Ancient Sun, 463-473 (1980).

33. Kerridge, J. F., Rev. Geophys. 31, 423-437 (1993).

34. Geiss, J. and Bochsler P., Geochim.Cosmochim. Acta 46, 529-548 (1982).

35. Wimmer-Schweingruber, R. F., Bochsler, P., and Kern, O., J. Geophys. Res. 103, 20621-20630 (1998).

36. Kallenbach, R., AIP Conf. Proc. this volume (2001).

37. Wieler, R., Humbert, F., and Marty, B., Earth Planet. Sci. Lett. 167, 47-60 (1999).

38. Kim, J. S., Kim, Y., Marti, K., and Kerridge, J. F., Nature 375, 383-385 (1995).

39. Hashizume, K., Chaussidon, M., Marty, B., and Robert, F., Science 290, 1142-1145 (2000).

40. Hashizume, K., Marty, B., and Wieler, R., Lunar Planet. Sci. Conf. XXX, \#1567 CD-ROM (1999).

41. Hashizume, K., Marty, B., Chaussidon, M., and Robert, F., AIP Conf. Proc. this volume (2001).

42. Kallenbach, R., Geiss, J., Ipavich, F. M., Gloeckler, G., Bochsler, P., Gliem, F., Hefti, S., Hilchenbach, M., and Hovestadt, D., Astrophys. J. 507, L85-L88 (1998).

43. Owen, T., Mahaffy P. R., Niemann, H. B., Atreya, S., and Wong M., Astrophys. J. Lett. 553, L77-L79 (2001).
44. Fouchet, T., Lellouch, E., Bézard, B., Encrenaz, T., Drossart, P., Feuchtgruber, H., and de Graauw, T., Icarus 143, 222-243 (2000).

45. Terzieva, R., and Herbst, E., MNRAS 317, 563-568 (2000).

46. Kerridge, J. F., AIP Conf. Proc. 341, 167-174 (1995).

47. Pepin, R. O., Icarus 92, 2-79 (1991).

48. Owen, T., Bar-Nun, A. , and Kleinfeld, I., Nature 358, 43-46 (1992).

49. Zahnle, K., "Planetary noble gases" in Protostars and planets III, edited by E. H. Levy and J. I. Lunine, Tucson: The University of Arizona Press, 1993, pp. 1305-1338.

50. Ozima, M., Wieler, R., Marty B., and Podosek F. A., Geochim. Cosmochim. Acta 62, 301-314 (1998).

51. Busemann, H., Baur, H., and Wieler, R., Meteorit. Planet. Sci. 35, 949-973 (2000).

52. Farley, K. A., and Neroda, E., Ann. Rev. Earth Sci. 26, 189-218 (1998).

53. Caffee, M. W., Hudson, G. B., Velsko, C., Huss, G. R., Alexander Jr., E. C., Chivas, A. R., Science 285, 21152118 (1999).

54. Swindle, T. D., AIP Conf. Proc. 341, 175-185 (1995).

55. Marti, K., and Mathew, K. J., Proc. Indian Acad. Sci. (Earth Planet. Sci.) 107, 425-431 (1998).

56. Pepin, R. O., Space Sci. Rev. 92, 371-395 (2000).

57. Busemann, H., and Eugster, O., Meteorit. Planet. Sci. 35, A37 (2000).

58. Zahnle, K., Pollack, J. B., and Kasting, J. K., Geochim. Cosmochim. Acta 54, 2577-2586 (1990).

59. Mahaffy, P. R., Niemann, H. B., Alpert, A., Atreya, S. K., Demick, J., Donahue, T. M., Harpold, D. N., and Owen, T. C., J. Geophys. Res. 105, 15061-15071 (2000).

60. Mahaffy, P. R., pers. comm. to R. Wieler (2001).

61. Eberhardt, P., Geiss, J., Graf, H., Grögler, N., Mendia, M. D., Mörgeli, M., Schwaller, H., Stettler, A., Krähenbühl, U., and v. Gunten, H. R., Proc. 3rd Lunar Sci. Conf., 1821-1856 (1972).

62. Wieler, R., and Baur, H., Meteoritics 29, 570-580 (1994).

63. Zinner, E., Annu. Rev. Earth Planet. Sci. 26, 147-188 (1998).

64. Hoppe, P., and Zinner, E., J. Geophys. Res. 105, 1037110385 (2000). 
65. Hoppe, P., AIP Conf. Proc. this volume (2001).

66. Hoppe, P., and Ott, U., AIP Conf. Proc. 402, 27-58 (1997).

67. Lequeux, J., J. Geophys. Res. 105, 10249-10255 (2000).

68. Nittler, L. R., Alexander, C. M. O'D., and Wang, J., Nature 393, 222 (1998).

69. Shukolyukov, A., and Lugmair, G. W., Space Sci. Rev. 92, 225-236 (2000).

70. Nyquist, L., Lindstrom, D., Mittlefehldt, D., Shih, C.-Y., Wiesmann, H., Wentworth, S., and Martinez, R., Meteorit. \& Planet. Sci. 36, 911-938 (2001).

71. Manuel, O. K., and Hwaung, G., Meteoritics 18, 209-222 (1983).

72. Bochsler, P., Ipavich, F. M., Paquette, J. A., Weygand, J. M., and Wurz, P., J. Geophys. Res. 105, 12659-12666 (2000).

73. Holweger, H., AIP Conf. Proc. this volume (2001).

74. Gloeckler G., and Geiss, J., Space Sci. Rev. 86, 127-159 (1998).

75. Kallenbach, R., Geiss, J., Gloeckler, G., and von Steiger, R., Astrophys. Space Sci. 274, 97-114 (2000).

76. Geiss, J., Gloeckler, G., Fisk, L. A., and von Steiger, R., J. Geophys. Res. 100, 23373 - 23377 (1995).

77. Gloeckler, G., Fisk, L. A., Geiss, J., Schwadron, N. A., and Zurbuchen, T. H., J. Geophys. Res. 105, 7459-7463 (2000).

78. Gloeckler, G., and Geiss, J., AIP Conf. Proc. this volume (2001).

79. Gloeckler, G., Fisk, L. A., Zurbuchen, T. H., Möbius, E., Funsten, H. O., Witte, M., and Roelof, E. C. EOS, Trans. Am. Geophys. Union 80, S237 (1999).

80. Baranov, V. B., Space Sci. Rev. 52, 89-120 (1990).

81. Fahr, H. J., Space Sci. Rev. 78, 199-212 (1996).

82. Gloeckler, G., Fisk, L. A., and J. Geiss, Nature 386, 374377 (1997).

83. Möbius, E., et al., Adv. Space Res. in press (2001).

84. Mewaldt, R. A., Liewer, P. C., and the Interstellar Probe Science and Technology Definition Team, Adv. Space Res. in press (2001).

85. Busemann, H., Baur, H., Wieler, R., Lunar Planet. Sci. Conf. XXXII, \#1598 CD-ROM (2000).
86. Gloeckler G., and Geiss, J., Space Sci. Rev. 84 275-284 (1998).

87. Salerno, E., Bühler, F., Bochsler, P., Busemann, H., Eugster, O., Zastenker, G. N., Agafonov, Y. N., and Eismont, N. A., AIP Conf. Proc. this volume (2001).

88. Gloeckler, G., and Geiss, J., in The Light Elements and Their Evolution, IAU Symp., edited by L. da Silva, M. Spite, and J. R. de Medeiros, 2000, Vol. 198, pp. 224233.

89. Klecker, B., Adv. Space Res. 23, 521-530 (1999).

90. Reames, D. V., Astrophys. J. 518, 473-479 (1999).

91. Cummings, A. C., Stone, E. C., and Steenberg, C. D., Proc. $26^{\text {th }}$ Int. Cosmic Ray Conf. 7, $531-534$ (1999).

92. Slavin, J. D., and Frisch, P. C., Astrophy. J., in press (2001).

93. Leske, R. A., AIP Conf. Proc. 516, 274-282 (2000).

94. Biswas, S., Space Sci. Rev. 75, 423-451 (1996).

95. Mazur, J. E., Mason, G. M., Blake, J. B., Klecker, B., Leske, R. A., Looper, M. D., and Mewaldt, R. A., J. Geophys. Res. 105, 21015-21023 (2000).

96. Möbius, E., Gloeckler, G., Fisk, L. A., and Mewaldt, R. A. "To the boundaries of the heliosphere and beyond" in The Outer Heliosphere: Beyond the Planets; edited by K. Scherer, H. Fichtner, E. Marsch, Katlenburg-Lindau, Copernicus Gesellschaft e.V., 2000, pp. 357-393.

97. Liewer, P. C., Mewaldt, R. A., Ayon, J. A., and Wallace, R. A., AIP Conf. Proc. 504, 911 (2000).

98. Engelmann, J. J., Ferrando, P., Soutoul, A., Goret, P., Juliusson, E., Koch-Miramond, L., Lund, N., Masse, P., Peters, B., Petrou, N., and Rasmussen, I. L., Astron. Astrophys. 233, 96-111 (1990).

99. DuVernois, M. A., Simpson, J. A., and Thayer, M. R., Astron. Astrophys. 316, 555-563 (1996).

100. Leske, R. A., Astrophys. J. 405, 567-583 (1993).

101. Garcia-Munoz, M., and Simpson, J. A., Proc. $16^{\text {th }}$ Int. Cosmic Ray Conf. 1, 270-275 (1979).

102. Wiedenbeck, M. E., Binns, W. R., Christian, E. R., Cummings, A. C., Dougherty, B. L., Hink, P. L., Klarmann, J., Leske, R. A., Lijowski, M., Mewaldt, R. A., Stone, E. C., Thayer, M. R., Von Rosenvinge, T. T., and Yanasak, N. E. , Astrophys. J. Lett., 523, L61-L64 (1999).

103. Meneguzzi, M., Audouze, J., and Reeves, H. Astron. Astrophys. 15, 337-359 (1971). 
104. Yanasak, N. E., Wiedenbeck, M. E., Mewaldt, R. A., Davis, A. J., Cummings, A. C., George, J. S., Leske, R. A., Stone, E. C., Christian, E. R., von Rosenvinge, T. T., Binns, W. R., Hink, P. L., and Israel, M. H., submitted to Astrophys. J. (2001).

105. Connell, J. J., Astrophys. J. Lett. 501, L59-L62, (1998).

106. Yanasak, N. E., Wiedenbeck, M. E., Binns, W. R., Christian, E. R., Cummings, A. C., Davis, A. J., George, J. S., Hink, P. L., Israel, M. H., Leske, R. A., Lijowski, M., Mewaldt, R. A., Stone, E. C., von Rosenvinge, T. T., Adv. Space Res. 27, 727-736 (2001).

107. Strong, A. W., and Moskalenko, I. V., Adv. Space Res. 27, 717-726 (2001).

108. Webber, W. R., and Kish, J., Proc. $16^{\text {th }}$ Int. Cosmic Ray Conf. 1, 389-394 (1979).

109. Ahlen, S. P., Greene, N. R., Loomba, D., Mitchel, J. W., Bower, C. R., Heinz, R. M., Mufson, S. L., Musser, J., Pitts, J. J., Spiczak, G. M., Clem, J., Guzik, T. G., Lijowski, M., Wefel, J. P., McKee, S., Nutter, S., Tomasch, A., Beatty, J. J., Ficenec, D. J., and Tobias, S., Astrophys. J. 534, 757-769 (2000).

110. Hams, T., Barbier, L. M., Bremerich, M., Christian, E. R., de Nolfo, G. A., Geier, S., Goebel, H., Gupta, S. K., Hof, M., Menn, W., Mewaldt, R. A., Mitchell, J. W., Schindler, S. M., Simon, M. and Streitmatter, R. E., to be published in Proc. $27^{\text {th }}$ Int. Cosmic Ray Conf. (2001).

111. de Nolfo, G. A., Barbier, L. M., Bremerich, M., Christian, E. R., Davis, A. J., Geier, S., Goebel, H., Gupta, S. K., Hams, T., Hof, M., Menn, W., Mewaldt, R. A., Mitchell, J. W., Schindler, S. M., Simon, M. and Streitmatter, R. E., to be published in Proc. $27^{\text {th }}$ Int. Cosmic Ray Conf. (2001).

112. Binns, W. R., Garrard, T. L., Gibner, P. S., Israel, M. H., Kertzman, M. P., Klarmann, J., Newport, B. J., Sotne, E. C., and Waddington, C. J., Astrophys. J. 346, 997-1009 (1989).

113. Westphal, A. J., Price, P. B., Weaver, B. A., and Afanasiev, V. G., Nature 396, 50-52 (1998).

114. Meyer, J.-P., Drury L. O'C., and Ellison, D. C., Space Sci. Rev. 86, 179-201 (1988).

115. Meyer, J.-P., Astrophys. J. Suppl. Ser. 57, 173-204 (1985).

116. Binns, W.R., Adams, J.H., Barbier, L.M., Christian, E.R., Craig, N. , Cummings, A.C., Cummings, J.R., Doke, T., Hasebe, N., Hayashi, T., Israel, M.H., Lee, D., Leske, R.A., Mark, D., Mewaldt, R.A., Mitchell, J.W., Ogura, K., Schindler, S.M., Stone, E.C., Tarlé, G., Tawara, H., Waddington, C.J., Westphal, A.J., Wiedenbeck, M.E., Yasuda, N., to be published in Proc. $27^{\text {th }}$ Int. Cosmic Ray Conf. (2001).
117. Higdon, J. C., Lingenfelter, R. E., and Ramaty, R., Astrophys. J .509, L33-L36 (1988).

118. Clayton, D. D., Astrophys. J. 334, 191-195 (1988).

119. Timmes, F. X., and Clayton, D. D., Astrophys. J. 472, 723-741 (1996).

120. Lugaro, M., Zinner, E., Gallino, R., and Amari, S., Astrophys. J. 527, 369-394 (1999).

121. Romano D., Matteucci, F., Molaro, P., and Bonifacio, P., Astron. Astrophys. 352, 117-128 (1999).

122. Bonifacio, P., and Molaro, P., MNRAS 285, 847-861 (1997).

123. Spite, F., and Spite, M., Astron. Astrophys. 115, 357366 (1982).

124. Romano, D., Matteucci, F., Ventura, P., D'Antona, F., Astron. Astrophys. 374, 646-655 (2001).

125. Pagel, B. E. J., Nucleosynthesis and chemical evolution of galaxies, Cambridge: Cambridge University Press, 1997.

126. Ryan, S. G., Beers T. C., Olive K. A., Fields, B. D., and Norris, J. E. Astrophys. J. 530, L57-L60 (2000).

127. Burles, S., Nollett, K. M., and Turner, M. S., Astrophys. J. 552, L1-L5 (2001)

128. Chiappini, C., Matteucci, F., and Gratton, R., Astrophys. J. 477, 765-780 (1997).

129. Chiappini, C., and Matteucci, F., AIP Conf. Proc. this volume (2001).

130. Chiappini, C., Matteucci, F., and Romano, D., Astrophys. J. 554, 1044-1058 (2001).

131. Charbonnel, C., and do Nascimento Jr., J. D., Astron. Astrophys. 336, 915-919 (1998).

132. Tytler, D.,O'Meara, J. M., Suzuki, N., and Lubin, D., Physica Scripta T85, 12-31 (2000).

133. O’Meara, J. M., Tytler, D., Kirkman, D., Suzuki, N., Prochaska, J. X., Lubin, D., and Wolfe, A. M., Astrophys. J. 552, 718-730 (2001).

134. Linsky, J. L., Space Sci. Rev. 84, 285-296 (1998).

135. Geiss, J., and Gloeckler, G., Space Sci. Rev. 84, 239250 (1998).

136. Sonneborn, G., Tripp, G. M., Ferlet, R., Jenkins, E. B., Sofia, U. J., Vidal-Madjar, A., Wozniak, R., Astrophys. J. $545277-289$ (2000).

137. Tosi, M., Steigman, G., Matteucci, F., and Chiappini, C., Astrophys. J. 498, 226-235 (1998). 
138. Chiappini, C., and Matteucci, F., in The Light Elements and Their Evolution, IAU Symp., edited by L. da Silva, M. Spite, and J. R. de Medeiros (2000), Vol. 198, pp. 540-546.

139. Bania, T. M., Rood, R. T., and Balser, D. S., in The Light Elements and Their Evolution, IAU Symp., edited by L. da Silva, M. Spite, and J. R. de Medeiros, 2000, Vol. 198, pp. 214-223.

140. Viegas, S. M., Gruenwald, R., and Steigman, G., Astrophys. J. 531, 813-819 (2000).

141. Maciel, W. J., in The Chemical Evolution of the Milky Way, edited by F. Matteucci and F. Giovanelli, Dordrecht: Kluwer, 2001, pp. 81-92.

142. Pagel, B. E. J., Simonson, E. A., Terlevich, R. J., and Edmunds, M. G., MNRAS 255, 325-345 (1992).

143. Izotov, Y., and Thuan, T. X., Astrophys. J. 500, 188216 (1998).

144. Kirilova, D., AIP Conf. Proc. this volume (2001).

145. Kirilova D., and Chizhov M., Nucl. Phys. B (Proc. Suppl.) 100, 360-362 (2001).

146. Tosi, M., "The chemical evolution of $\mathrm{D}$ and ${ }^{3} \mathrm{He}$ in the Galaxy in connection with CNO elements" in The Chemical Evolution of the Milky Way, edited by F. Matteucci and F. Giovanelli, Dordrecht: Kluwer, 2001, pp. 505-515.

147. Charbonnel, C., Brown, J. A., and Wallerstein, G., Astron. Astrophys.. 332, 204-214 (1998).

148. Boothroyd, A. I., and Sackmann, I.-J., Astrophys. J. 510, 232-250 (1999).

149. Ryan, S. G., Kajino, T., Beers, T. C., Suzuki, T. K., Romano, D., Matteucchi, F., Rosolankova K., Astrophys. J. 549, 55-71 (2001). 